Supporting information for:

\title{
Highly Stereoselective Aldol Reaction Based on Titanium Enolates from $(S)$-1-Benzyloxy-2-methyl-3-pentanone
}

Joan G. Solsona, Joaquim Nebot, Pedro Romea,* Fèlix Urpí.*

1. General Experimental Methods. $\quad$ S2

2. Experimental Procedures Corresponding to Table 1. S2

3. Characterization data for aldol diastereomers. S3

$(2 S, 4 R, 5 S)$-1-Benzyloxy-5-hydroxy-2,4,7-trimethyl-3-octanone (4b) S3

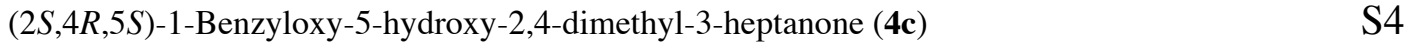

$(1 R, 2 R, 4 S)$-5-Benzyloxy-1-hydroxy-2,4-dimethyl-1-phenyl-3-pentanone (4d) S4

$(2 S, 4 R, 5 R)$-1-Benzyloxy-5-hydroxy-2,4,6-trimethyl-6-hepten-3-one (4e) S5

$(2 S, 4 R, 5 S, 6 E)$-1-Benzyloxy-5-hydroxy-2,4-dimethyl-6-octen-3-one (4f) S5

(2S,4R,5S,6S)-1-Benzyloxy-7-(tert-butyldiphenylsilyloxy)-5-hydroxy-

2,4,6-trimethyl-3-heptanone (8)

S6

(2S,4R,5S,6R)-1-Benzyloxy-7-(tert-butyldiphenylsilyloxy)-5-hydroxy-

2,4,6-trimethyl-3-heptanone (9)

S6

(2S,4R,5R,6R)-1-Benzyloxy-6-(tert-butyldiphenylsilyloxy)-5-hydroxy-

$\begin{array}{ll}\text { 2,4-dimethyl-3-heptanone (10) } & \text { S7 }\end{array}$

4. Stereochemical proof for $\mathbf{4 a} \quad$ S8

$(2 S, 4 R, 5 S)$-1,5-Dihydroxy-2,4,6-trimethyl-3-heptanone (12) S8

5. Stereochemical proof for $11 \quad$ S9

(2S,3R,4S,5R,6S)-1-Benzyloxy-6-(tert-butyldiphenylsilyloxy)-3,5-O-isopropylidene-

2,4-dimethyl-3,5-heptanediol (13) S9

$(3 R, 4 S, 5 R, 6 S)$-7-Benzyloxy-3,5-isopropylidenedioxy-4,6-dimethyl-2-heptanone (14) S10

6. ${ }^{1} \mathrm{H}$ and ${ }^{13} \mathrm{C}$ spectra of aldol diastereomers, diol 12 and ketone $14 \quad \mathrm{~S} 11$ 


\section{General Experimental Methods}

Specific rotations were determined at $20{ }^{\circ} \mathrm{C}$. Only the more representative frequencies $\left(\mathrm{cm}^{-1}\right)$ observed in IR spectra are reported. ${ }^{1} \mathrm{H}$ NMR and ${ }^{13} \mathrm{C}$ NMR spectra were recorded at $25{ }^{\circ} \mathrm{C}$. Chemical shifts $(\delta)$ are quoted in ppm and referenced to internal TMS $(\delta 0)$ or DMSO $(\delta 2.5)$ for ${ }^{1} \mathrm{H}$ NMR, and $\mathrm{CDCl}_{3}(\delta 77.0)$ or DMSO- $d_{6}(\delta 39.5)$ for ${ }^{13} \mathrm{C}$ NMR; coupling constants $(J)$ are quoted in $\mathrm{Hz}$; data are reported as follows: s, singlet; d, doublet; t, triplet; q, quartet; m, multiplet; br, broad; where appropriate, 2D techniques were also used to assist in structure elucidation. HPLC analyses were performed with a Tracer Spherisorb S3W column $\left(4.6 \mathrm{~mm} \times 25 \mathrm{~cm}, 3 \mathrm{~mm}\right.$ silica gel, $\left.0.9 \mathrm{~mL} \mathrm{~min}{ }^{-1}\right)$. Flash chromatography was performed on silica gel $60(35-70 \mu \mathrm{m})$.

All reactions were conducted in oven-dried glassware under inert atmosphere of nitrogen with anhydrous solvents. The solvents and reagents were purified and dried according to standard procedures.

\section{Experimental Procedures Corresponding to Table 1.}

\section{Experimental procedure A. PG: Bn, TBDPS; Lewis acid: $\mathrm{TiCl}_{4}$}

$\mathrm{TiCl}_{4}(120 \mu \mathrm{L}, 1.1 \mathrm{mmol})$ was added dropwise to a solution of ketone $\mathbf{1}$ or $\mathbf{3}(1.0 \mathrm{mmol})$ in $\mathrm{CH}_{2} \mathrm{Cl}_{2}(5$ $\mathrm{mL})$ at $-78{ }^{\circ} \mathrm{C}$ under $\mathrm{N}_{2}$. The orange solution was stirred for $2 \mathrm{~min}$ and $i-\mathrm{Pr}_{2} \mathrm{NEt}(190 \mu \mathrm{L}, 1.1 \mathrm{mmol})$ was added dropwise. The resulting dark red solution was stirred for $1.5 \mathrm{~h}$ at $-78{ }^{\circ} \mathrm{C}$ and isobutyraldehyde $(140 \mu \mathrm{L}, 1.5 \mathrm{mmol})$ was added. After $2 \mathrm{~h}$ at $-78{ }^{\circ} \mathrm{C}$ the reaction was quenched by addition of saturated $\mathrm{NH}_{4} \mathrm{Cl}(5 \mathrm{~mL})$ and vigorously stirred at room temperature. The mixture was diluted with $\mathrm{Et}_{2} \mathrm{O}$ and washed with $\mathrm{H}_{2} \mathrm{O}$, saturated $\mathrm{NaHCO}_{3}$, and brine. The aqueous layers were extracted with $\mathrm{Et}_{2} \mathrm{O}$ and the combined extracts were dried $\left(\mathrm{MgSO}_{4}\right)$ and concentrated. The resulting oil was analyzed by HPLC and purified by flash chromatography on silica gel.

Experimental procedure B. PG: Bn, TBDPS; Lewis acid: $\mathrm{TiCl}_{4}(2$ eq)

$\mathrm{TiCl}_{4}(120 \mu \mathrm{L}, 1.1 \mathrm{mmol})$ was added dropwise to a solution of $\mathbf{1}$ or $\mathbf{3}(1.0 \mathrm{mmol})$ in $\mathrm{CH}_{2} \mathrm{Cl}_{2}(5 \mathrm{~mL})$ at $-78{ }^{\circ} \mathrm{C}$ under $\mathrm{N}_{2}$. The orange solution was stirred for $2 \mathrm{~min}$ and $i-\mathrm{Pr}_{2} \mathrm{NEt}(190 \mu \mathrm{L}, 1.1 \mathrm{mmol})$ was 
added dropwise. The resulting dark red solution was stirred for $1.5 \mathrm{~h}$ at $-78{ }^{\circ} \mathrm{C}$ and additional $\mathrm{TiCl}_{4}$ (110 $\mu \mathrm{L}, 1.0 \mathrm{mmol}$ ) were added dropwise. The solution was stirred for $10 \mathrm{~min}$ and isobutyraldehyde $(140 \mu \mathrm{L}, 1.5 \mathrm{mmol})$ was added. After $2 \mathrm{~h}$ at $-78{ }^{\circ} \mathrm{C}$ the reaction was quenched by addition of saturated $\mathrm{NH}_{4} \mathrm{Cl}(5 \mathrm{~mL})$ and vigorously stirred at room temperature. The mixture was diluted with $\mathrm{Et}_{2} \mathrm{O}$ and washed with $\mathrm{H}_{2} \mathrm{O}$, saturated $\mathrm{NaHCO}_{3}$, and brine. The aqueous layers were extracted with $\mathrm{Et}_{2} \mathrm{O}$ and the combined extracts were dried $\left(\mathrm{MgSO}_{4}\right)$ and concentrated. The resulting oil was analyzed by HPLC and purified by flash chromatography on silica gel.

\section{Experimental procedure C. PG: Bn, PMB, or TBDPS; Lewis acid: (i-PrO)TiCl ${ }_{3}$}

Freshly distilled $(i \text {-PrO })_{4} \mathrm{Ti}(83 \mu \mathrm{L}, 0.28 \mathrm{mmol})$ was added dropwise to a solution of $\mathrm{TiCl}_{4}(92 \mu \mathrm{L}$, $0.84 \mathrm{mmol})$ in $\mathrm{CH}_{2} \mathrm{Cl}_{2}(1 \mathrm{~mL})$ at $0{ }^{\circ} \mathrm{C}$ under $\mathrm{N}_{2}$. The mixture was stirred for 10 min at $0{ }^{\circ} \mathrm{C}$ and 10 min at room temperature, diluted with $\mathrm{CH}_{2} \mathrm{Cl}_{2}(1 \mathrm{~mL})$, and the resulting colorless solution was added via cannula or syringe $(2 \times 0.5 \mathrm{~mL})$ to a solution of $\mathbf{1}, \mathbf{2}$, or $\mathbf{3}(1.0 \mathrm{mmol})$ in $\mathrm{CH}_{2} \mathrm{Cl}_{2}(2 \mathrm{~mL})$ at $-78{ }^{\circ} \mathrm{C}$ under $\mathrm{N}_{2}$. The pale yellow solution was stirred for $2 \mathrm{~min}$ and $i-\operatorname{Pr}_{2} \mathrm{NEt}(190 \mu \mathrm{L}, 1.1 \mathrm{mmol})$ was added dropwise. The resulting colored solution was stirred for $1.5 \mathrm{~h}$ at $-78^{\circ} \mathrm{C}$ and isobutyraldehyde $(140$ $\mu \mathrm{L}, 1.5 \mathrm{mmol}$ ) was added. After $2 \mathrm{~h}$ at $-78{ }^{\circ} \mathrm{C}$ the reaction was quenched by addition of saturated $\mathrm{NH}_{4} \mathrm{Cl}(5 \mathrm{~mL})$ and vigorously stirred at room temperature. The mixture was diluted with $\mathrm{Et}_{2} \mathrm{O}$ and washed with $\mathrm{H}_{2} \mathrm{O}$, saturated $\mathrm{NaHCO}_{3}$, and brine. The aqueous layers were extracted with $\mathrm{Et}_{2} \mathrm{O}$ and the combined extracts were dried $\left(\mathrm{MgSO}_{4}\right)$ and concentrated. The resulting oil was analyzed by HPLC and purified by flash chromatography on silica gel.

\section{Characterization data for aldol diastereomers.}

$(2 S, 4 R, 5 S)$-1-Benzyloxy-5-hydroxy-2,4,7-trimethyl-3-octanone (4b).

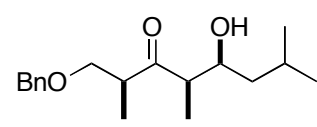

Colorless oil. $R_{f}\left(\right.$ hexanes/EtOAc 90:10) $=0.2 ;$ HPLC $\left(\right.$ hexanes $/ i$-PrOH 99.5:0.5) $t_{\mathrm{R}}=16.3 \mathrm{~min} ;[\alpha]_{\mathrm{D}}=$ +5.0 (c 1.14, $\mathrm{CHCl}_{3}$ ); IR (film): v 3500, 3031, 2956, 2870, 1706, 1456, 1367, $1095 \mathrm{~cm}^{-1}$; ${ }^{1} \mathrm{H}$ NMR $\left(400 \mathrm{MHz}, \mathrm{CDCl}_{3}\right) \delta$ 7.35-7.25 (5H, m), $4.46(1 \mathrm{H}, \mathrm{AB}$ system, $J=11.9), 4.44(1 \mathrm{H}, \mathrm{AB}$ system, $J=$ 
11.9), $4.10(1 \mathrm{H}$, ddd, $J=9.4, J=4.1, J=2.7), 3.65(1 \mathrm{H}, \mathrm{t}, J=8.8), 3.46(1 \mathrm{H}, \mathrm{dd}, J=8.8, J=4.9)$, 3.22-3.13 (1H, m), $2.71(1 \mathrm{H}, \mathrm{qd}, J=7.2, J=2.7), 1.75-1.65(1 \mathrm{H}, \mathrm{m}), 1.43(1 \mathrm{H}, \mathrm{ddd}, J=13.7, J=$ 9.4, $J=5.7), 1.09$ ( $1 \mathrm{H}$, ddd, $J=13.7, J=8.6, J=4.1), 1.08$ (3H, d, $J=7.2), 1.02(3 \mathrm{H}, \mathrm{d}, J=7.0$ ), $0.88(3 \mathrm{H}, \mathrm{d}, J=6.6), 0.87(3 \mathrm{H}, \mathrm{d}, J=6.6) ;{ }^{13} \mathrm{C} \mathrm{NMR}\left(100.6 \mathrm{MHz}, \mathrm{CDCl}_{3}\right) \delta 218.2(\mathrm{C}), 137.5(\mathrm{C})$, $128.4(\mathrm{CH}), 127.8(\mathrm{CH}), 127.6(\mathrm{CH}), 73.4\left(\mathrm{CH}_{2}\right), 73.2\left(\mathrm{CH}_{2}\right), 68.5(\mathrm{CH}), 51.2(\mathrm{CH}), 44.8(\mathrm{CH}), 42.6$ $\left(\mathrm{CH}_{2}\right), 24.6(\mathrm{CH}), 23.3\left(\mathrm{CH}_{3}\right), 22.0\left(\mathrm{CH}_{3}\right), 13.6\left(\mathrm{CH}_{3}\right), 9.0\left(\mathrm{CH}_{3}\right)$; HRMS (+FAB): $\mathrm{m} / z$ calcd. for $\mathrm{C}_{18} \mathrm{H}_{29} \mathrm{O}_{3}[\mathrm{M}+\mathrm{H}]^{+}:$293.2117. Found: 293.2110.

\section{$(2 S, 4 R, 5 S)-1-B e n z y l o x y-5-h y d r o x y-2,4-d i m e t h y l-3-h e p t a n o n e ~(4 c) .{ }^{1}$}

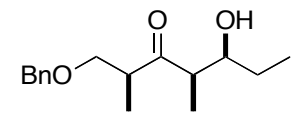

Colorless oil. $R_{f}\left(\right.$ hexanes $/$ EtOAc 85:15) $=0.2$; HPLC $($ hexanes $/ i-\operatorname{PrOH} 99.5: 0.5) t_{\mathrm{R}}=20.3 \mathrm{~min} ;[\alpha]_{\mathrm{D}}=$ $+15.2\left(c\right.$ 1.54, $\left.\mathrm{CHCl}_{3}\right)\left[\right.$ lit. $^{1}$ ent $-4 \mathrm{c}[\alpha]_{\mathrm{D}}=-16.2\left(c=1.8, \mathrm{CHCl}_{3}\right)$ ]; IR (film): v 3479, 3032, 2968, 2934, 1706, 1456, 1373, $1099 \mathrm{~cm}^{-1} ;{ }^{1} \mathrm{H}$ NMR (400 MHz, $\left.\mathrm{CDCl}_{3}\right) \delta$ 7.36-7.25 (5H, m), $4.48(1 \mathrm{H}, \mathrm{AB}$ system, $J=12.0), 4.44(1 \mathrm{H}, \mathrm{AB}$ system, $J=12.0), 3.91(1 \mathrm{H}, \mathrm{ddd}, J=8.4, J=5.4, J=2.7), 3.65(1 \mathrm{H}$, t, $J=8.8), 3.47(1 \mathrm{H}, \mathrm{dd}, J=8.8, J=4.6), 3.22-3.13(1 \mathrm{H}, \mathrm{m}), 2.76(1 \mathrm{H}, \mathrm{qd}, J=7.0, J=2.7)$, $1.55-1.44(1 \mathrm{H}, \mathrm{m}), 1.42-1.32(1 \mathrm{H}, \mathrm{m}), 1.07(3 \mathrm{H}, \mathrm{d}, J=7.0), 1.02(3 \mathrm{H}, \mathrm{d}, J=7.0), 0.90(3 \mathrm{H}, \mathrm{t}, J=$ 7.4); ${ }^{13} \mathrm{C}$ NMR (100.6 MHz, $\left.\mathrm{CDCl}_{3}\right) \delta 218.2(\mathrm{C}), 137.5(\mathrm{C}), 128.4(\mathrm{CH}), 127.8(\mathrm{CH}), 127.7(\mathrm{CH})$, $73.4\left(\mathrm{CH}_{2}\right), 73.2\left(\mathrm{CH}_{2}\right), 72.1(\mathrm{CH}), 50.4(\mathrm{CH}), 44.8(\mathrm{CH}), 26.5\left(\mathrm{CH}_{2}\right), 13.6\left(\mathrm{CH}_{3}\right), 10.5\left(\mathrm{CH}_{3}\right), 8.7$ $\left(\mathrm{CH}_{3}\right)$; HRMS (+FAB): $\mathrm{m} / z$ calcd. for $\mathrm{C}_{16} \mathrm{H}_{25} \mathrm{O}_{3}[\mathrm{M}+\mathrm{H}]^{+}:$265.1804. Found: 265.1811 .

\section{$(1 R, 2 R, 4 S)$-5-Benzyloxy-1-hydroxy-2,4-dimethyl-1-phenyl-3-pentanone (4d).}

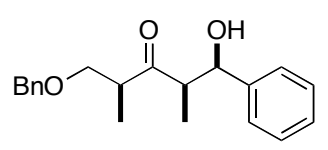

Colorless oil. $R_{f}\left(\right.$ hexanes/EtOAc 90:10) $=0.2 ;$ HPLC $\left(\right.$ hexanes $/ i$-PrOH 99.5:0.5) $t_{\mathrm{R}}=12.7 \mathrm{~min} ;[\alpha]_{\mathrm{D}}=$ +13.3 (c 1.58, $\mathrm{CHCl}_{3}$ ); IR (film): $v$ 3484, 3031, 2934, 2875, 1702, 1496, 1453, 1365, $1098 \mathrm{~cm}^{-1} ;{ }^{1} \mathrm{H}$ NMR (400 MHz, $\left.\mathrm{CDCl}_{3}\right)$ d 7.37-7.20 (10H, m), 5.19 (1H, d, $\left.J=2.9\right), 4.48$ (1H, AB system, $J=11.9$ ), $4.45(1 \mathrm{H}, \mathrm{AB}$ system, $J=11.9), 3.57(1 \mathrm{H}, \mathrm{t}, J=8.8), 3.49(1 \mathrm{H}, \mathrm{dd}, J=8.8, J=5.3), 3.16-3.08(1 \mathrm{H}$, 
m), $2.98(1 \mathrm{H}, \mathrm{qd}, J=7.2, J=2.9), 1.04(3 \mathrm{H}, \mathrm{d}, J=6.8), 0.98(3 \mathrm{H}, \mathrm{d}, J=7.2) ;{ }^{13} \mathrm{C} \mathrm{NMR}(100.6 \mathrm{MHz}$, $\left.\mathrm{CDCl}_{3}\right) \delta 218.5(\mathrm{C}), 141.6(\mathrm{C}), 137.6(\mathrm{C}), 128.5(\mathrm{CH}), 128.1(\mathrm{CH}), 127.8(\mathrm{CH}), 127.7(\mathrm{CH}), 127.0$ $(\mathrm{CH}), 125.8(\mathrm{CH}), 73.5\left(\mathrm{CH}_{2}\right), 73.2\left(\mathrm{CH}_{2}\right), 72.0(\mathrm{CH}), 52.9(\mathrm{CH}), 45.0(\mathrm{CH}), 13.3\left(\mathrm{CH}_{3}\right), 8.9\left(\mathrm{CH}_{3}\right)$; HRMS (+FAB): $m / z$ calcd. for $\mathrm{C}_{20} \mathrm{H}_{25} \mathrm{O}_{3}[\mathrm{M}+\mathrm{H}]^{+}: 313.1804$. Found: 313.1800 .

$(2 S, 4 R, 5 R)-1-B e n z y l o x y-5-h y d r o x y-2,4,6-t r i m e t h y l-6-h e p t e n-3-o n e ~(4 e){ }^{2}$

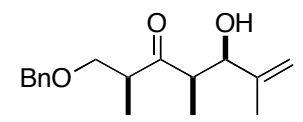

Colorless oil. $R_{f}\left(\right.$ hexanes/EtOAc 90:10) $=0.2$; HPLC $\left(\right.$ hexanes $/ i$-PrOH 99.5:0.5) $t_{\mathrm{R}}=15.3 \min ;[\alpha]_{\mathrm{D}}=$ $+45.9\left(c\right.$ 2.23, $\left.\mathrm{CHCl}_{3}\right)\left[\right.$ lit. $^{2}[\alpha]_{\mathrm{D}}=+43.6\left(c\right.$ 2.1, $\left.\left.\mathrm{CHCl}_{3}\right)\right]$; IR (film): v 3492, 3032, 2935, 2875, 1704 , 1653, 1454, 1373, $1098 \mathrm{~cm}^{-1} ;{ }^{1} \mathrm{H}$ NMR (400 MHz, $\left.\mathrm{CDCl}_{3}\right) \delta$ 7.35-7.26 (5H, m), 5.09 (1H, broad s), $4.93(1 \mathrm{H}$, broad q, $J=1.6), 4.51(1 \mathrm{H}$, broad s $), 4.49(1 \mathrm{H}, \mathrm{AB}$ system, $J=12.1), 4.45(1 \mathrm{H}, \mathrm{AB}$ system, $J=12.1), 3.62(1 \mathrm{H}, \mathrm{t}, J=8.8), 3.48(1 \mathrm{H}, \mathrm{dd}, J=8.8, J=5.1), 3.22-3.13(1 \mathrm{H}, \mathrm{m}), 2.87(1 \mathrm{H}, \mathrm{qd}, J=$ $7.2, J=2.5), 1.63\left(3 \mathrm{H}\right.$, broad s), $1.05(3 \mathrm{H}, \mathrm{d}, J=6.8), 1.02(3 \mathrm{H}, \mathrm{d}, J=7.2) ;{ }^{13} \mathrm{C} \mathrm{NMR}(100.6 \mathrm{MHz}$, $\left.\mathrm{CDCl}_{3}\right) \delta 218.1(\mathrm{C}), 143.3(\mathrm{C}), 137.6(\mathrm{C}), 128.4(\mathrm{CH}), 127.8(\mathrm{CH}), 127.7(\mathrm{CH}), 111.4\left(\mathrm{CH}_{2}\right), 73.5$ $\left(\mathrm{CH}_{2}\right), 73.1\left(\mathrm{CH}_{2}\right), 72.6(\mathrm{CH}), 48.4(\mathrm{CH}), 44.6(\mathrm{CH}), 19.6\left(\mathrm{CH}_{3}\right), 13.5\left(\mathrm{CH}_{3}\right), 8.2\left(\mathrm{CH}_{3}\right)$.

$(2 S, 4 R, 5 S, 6 E)-1-B e n z y l o x y-5-h y d r o x y-2,4-d i m e t h y l-6-o c t e n-3-o n e ~(4 f) .{ }^{2,3}$

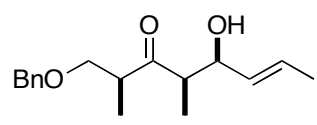

Colorless oil. $R_{f}$ (hexanes/EtOAc 90:10) $=0.2$; HPLC $\left(\right.$ hexanes $/ i$-PrOH 99.5:0.5) $t_{\mathrm{R}}=20.3 \min ;[\alpha]_{\mathrm{D}}=$ -2.1 (c 1.46, $\mathrm{CHCl}_{3}$ ) [lit. ${ }^{3}$ ent-4f $[\alpha]_{\mathrm{D}}=+3.5$ (c 4.5, $\left.\mathrm{CHCl}_{3}\right)$ ]; IR (film): $v$ 3474, 3031, 2935, 2876, 1706, 1455, 1374, $1097 \mathrm{~cm}^{-1} ;{ }^{1} \mathrm{H}$ NMR $\left(400 \mathrm{MHz}, \mathrm{CDCl}_{3}\right) \delta 7.37-7.26(5 \mathrm{H}, \mathrm{m}), 5.66(1 \mathrm{H}, \mathrm{dqd}, J=$ $15.2, J=6.4, J=1.2), 5.41(1 \mathrm{H}, \mathrm{ddq}, J=15.2, J=6.4, J=1.6), 4.49(1 \mathrm{H}$, AB system,$J=12.0), 4.47$ (1H, AB system, $J=12.0), 4.43-4.41(1 \mathrm{H}, \mathrm{m}), 3.64(1 \mathrm{H}, \mathrm{t}, J=8.8), 3.45(1 \mathrm{H}, \mathrm{dd}, J=8.8, J=5.2)$, 3.20-3.11 (1H, m), $2.84(1 \mathrm{H}, \mathrm{qd}, J=7.6, J=3.6), 1.67(3 \mathrm{H}, \mathrm{dd}, J=6.4, J=1.2), 1.08(3 \mathrm{H}, \mathrm{d}, J=$ 7.6), $1.03(3 \mathrm{H}, \mathrm{d}, J=7.2) ;{ }^{13} \mathrm{C}$ NMR (100.6 MHz, $\left.\mathrm{CDCl}_{3}\right) \delta 217.8(\mathrm{C}), 137.6(\mathrm{C}), 130.3(\mathrm{CH}), 128.4$ $(\mathrm{CH}), 127.8(\mathrm{CH}), 127.7(\mathrm{CH}), 127.6(\mathrm{CH}), 73.4\left(\mathrm{CH}_{2}\right), 72.9\left(\mathrm{CH}_{2}\right), 72.3(\mathrm{CH}), 51.3(\mathrm{CH}), 45.3(\mathrm{CH})$, 
$17.7\left(\mathrm{CH}_{3}\right), 13.5\left(\mathrm{CH}_{3}\right), 10.1\left(\mathrm{CH}_{3}\right)$; HRMS (+FAB): $m / z$ calcd. for $\mathrm{C}_{17} \mathrm{H}_{25} \mathrm{O}_{3}[\mathrm{M}+\mathrm{H}]^{+}: 277.1804$. Found: 277.1805.

\section{$(2 S, 4 R, 5 S, 6 S)$-1-Benzyloxy-7-(tert-butyldiphenylsilyloxy)-5-hydroxy-2,4,6-trimethyl-3-} heptanone (8).

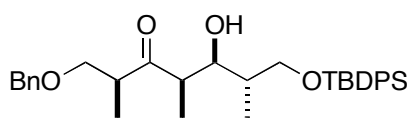

Colorless oil. $R_{f}\left(\right.$ hexanes/EtOAc 90:10) $=0.3$; HPLC $($ hexanes $/ i-\operatorname{PrOH} 99.5: 0.5) t_{\mathrm{R}}=10.4 \min ;[\alpha]_{\mathrm{D}}=$ $+4.4\left(c\right.$ 1.3, $\left.\mathrm{CHCl}_{3}\right)\left[\right.$ lit. $^{3}[\alpha]_{\mathrm{D}}=+3.1\left(c\right.$ 2.6, $\left.\left.\mathrm{CHCl}_{3}\right)\right]$; IR (film): v 3506, 3070, 2932, 2858, 1713, 1457, 1428, $1111 \mathrm{~cm}^{-1} ;{ }^{1} \mathrm{H}$ NMR (400 MHz, $\left.\mathrm{CDCl}_{3}\right) \delta$ 7.69-7.66 (4H, m), 7.44-7.35 (6H, m), 7.27-7.19 (5H, m), $4.44(1 \mathrm{H}, \mathrm{AB}$ system, $J=12.0), 4.40(1 \mathrm{H}$, AB system, $J=12.0), 4.03(1 \mathrm{H}, \mathrm{dd}, J=$ 9.0, $J=2.7), 3.79(1 \mathrm{H}, \mathrm{dd}, J=9.9, J=4.9), 3.71(1 \mathrm{H}, \mathrm{dd}, J=9.9, J=4.3), 3.63(1 \mathrm{H}, \mathrm{t}, J=8.8), 3.42$ (1H, dd $J=8.8, J=5.2), 3.21-3.12(1 \mathrm{H}, \mathrm{m}), 2.79$ (1H, qd, $J=7.0, J=2.7), 1.80-1.71(1 \mathrm{H}, \mathrm{m}), 1.10$ $(3 \mathrm{H}, \mathrm{d}, J=7.0), 1.06(9 \mathrm{H}, \mathrm{s}), 1.03(3 \mathrm{H}, \mathrm{d}, J=6.8), 0.92(3 \mathrm{H}, \mathrm{d}, J=6.8) ;{ }^{13} \mathrm{C} \mathrm{NMR}(100.6 \mathrm{MHz}$, $\left.\mathrm{CDCl}_{3}\right) \delta 217.2(\mathrm{C}), 137.6(\mathrm{C}), 135.6(\mathrm{CH}), 133.5(2 \times \mathrm{C}), 129.6(\mathrm{CH}), 128.4(\mathrm{CH}), 127.7(\mathrm{CH})$, $127.6(2 \times \mathrm{CH}), 73.4\left(\mathrm{CH}_{2}\right), 73.0\left(\mathrm{CH}_{2}\right), 72.4(\mathrm{CH}), 66.8\left(\mathrm{CH}_{2}\right), 48.7(\mathrm{CH}), 44.2(\mathrm{CH}), 37.5(\mathrm{CH})$, $26.9\left(\mathrm{CH}_{3}\right), 19.3(\mathrm{C}), 14.0\left(\mathrm{CH}_{3}\right), 13.8\left(\mathrm{CH}_{3}\right), 8.2\left(\mathrm{CH}_{3}\right)$; HRMS (+FAB): $\mathrm{m} / z$ calcd. for $\mathrm{C}_{33} \mathrm{H}_{45} \mathrm{O}_{4} \mathrm{Si}$ $[\mathrm{M}+\mathrm{H}]^{+}:$533.3087. Found: 533.3079.

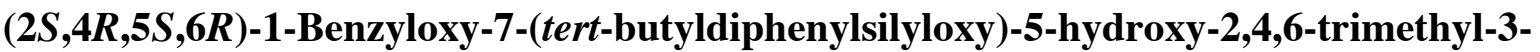
heptanone (9).

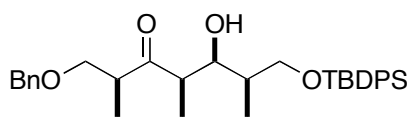

Colorless oil. $R_{f}\left(\right.$ hexanes/EtOAc 90:10) $=0.2$; HPLC $($ hexanes $/ i-\operatorname{PrOH} 99.5: 0.5) t_{\mathrm{R}}=14.0 \mathrm{~min} ;[\alpha]_{\mathrm{D}}=$ -3.0 (c 1.39, $\left.\mathrm{CHCl}_{3}\right)\left[\right.$ lit. $^{3}$ ent-9 $[\alpha]_{\mathrm{D}}=+3.6$ (c 2.2, $\left.\left.\mathrm{CHCl}_{3}\right)\right]$; IR (film): $v$ 3512, 3070, 2933, 2859, 1710, 1456, 1428, $1110 \mathrm{~cm}^{-1} ;{ }^{1} \mathrm{H}$ NMR (400 MHz, $\left.\mathrm{CDCl}_{3}\right) \delta$ 7.66-7.64 (4H, m), 7.45-7.36 (6H, m), 7.29-7.22 (5H, m), $4.43(1 \mathrm{H}, \mathrm{AB}$ system, $J=12.0), 4.40(1 \mathrm{H}$, AB system, $J=12.0), 4.07(1 \mathrm{H}, \mathrm{t}, J=$ 5.6), $3.66(1 \mathrm{H}, \mathrm{dd}, J=10.0, J=4.0), 3.62(1 \mathrm{H}, \mathrm{t}, J=8.8), 3.52(1 \mathrm{H}, \mathrm{dd}, J=10.0, J=4.2), 3.40(1 \mathrm{H}$, 
dd, $J=8.8, J=4.8), 3.13-3.01(1 \mathrm{H}, \mathrm{m}), 2.90(1 \mathrm{H}, \mathrm{qd}, J=6.8, J=5.6), 1.76-1.68(1 \mathrm{H}, \mathrm{m}), 1.11(3 \mathrm{H}$, d, $J=6.8), 1.06(9 \mathrm{H}, \mathrm{s}), 1.01(3 \mathrm{H}, \mathrm{d}, J=7.2), 0.97(3 \mathrm{H}, \mathrm{d}, J=6.8) ;{ }^{13} \mathrm{C} \mathrm{NMR}\left(100.6 \mathrm{MHz}, \mathrm{CDCl}_{3}\right) \delta$ $217.1(\mathrm{C}), 137.7(\mathrm{C}), 135.7(\mathrm{CH}), 135.5(\mathrm{CH}), 133.2(\mathrm{C}), 133.0(\mathrm{C}), 129.8(\mathrm{CH}), 129.7(\mathrm{CH}), 128.3$ $(\mathrm{CH}), 127.8(\mathrm{CH}), 127.7(\mathrm{CH}), 127.6(\mathrm{CH}), 73.5(\mathrm{CH}), 73.4\left(\mathrm{CH}_{2}\right), 72.8\left(\mathrm{CH}_{2}\right), 68.0\left(\mathrm{CH}_{2}\right), 49.4$ $(\mathrm{CH}), 45.2(\mathrm{CH}), 37.2(\mathrm{CH}), 26.8\left(\mathrm{CH}_{3}\right), 19.2(\mathrm{C}), 13.8\left(\mathrm{CH}_{3}\right), 12.2\left(\mathrm{CH}_{3}\right), 11.0\left(\mathrm{CH}_{3}\right) ; \mathrm{HRMS}$ (+FAB): $m / z$ calcd. for $\mathrm{C}_{33} \mathrm{H}_{45} \mathrm{O}_{4} \mathrm{Si}[\mathrm{M}+\mathrm{H}]^{+}:$533.3087. Found: 533.3077.

\section{(2S,4R,5R,6R)-1-Benzyloxy-6-(tert-butyldiphenylsilyloxy)-5-hydroxy-2,4-dimethyl-3-heptanone} (10).

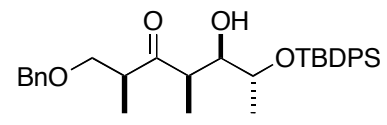

Colorless oil. $R_{f}$ (hexanes/EtOAc 90:10) $=0.25$; HPLC (hexanes $/ i$-PrOH 99.5:0.5) $t_{\mathrm{R}}=7.1 \mathrm{~min}$; IR (film): $v$ 3567, 3070, 2932, 2857, 1703, 1456, 1427, $1109 \mathrm{~cm}^{-1}$; ${ }^{1} \mathrm{H}$ NMR (400 MHz, $\mathrm{CDCl}_{3}$ ) 反 7.70-7.65 $(4 \mathrm{H}, \mathrm{m}), 7.45-7.25(11 \mathrm{H}, \mathrm{m}), 4.45(1 \mathrm{H}, \mathrm{AB}$ system, $J=12.0), 4.39(1 \mathrm{H}$, AB system, $J=$ 12.0), 3.85-3.79 (2H, m), 3.60 ( $1 \mathrm{H}, \mathrm{dd}, J=8.8, J=7.8$ ), 3.34 ( $1 \mathrm{H}, \mathrm{dd}, J=8.8, J=6.2$ ), 3.06-2.96 $(1 \mathrm{H}, \mathrm{m}), 2.79-2.72(1 \mathrm{H}, \mathrm{m}), 1.06(9 \mathrm{H}, \mathrm{s}), 0.99(3 \mathrm{H}, \mathrm{d}, J=6.0) ;{ }^{13} \mathrm{C} \mathrm{NMR}\left(100.6 \mathrm{MHz}, \mathrm{CDCl}_{3}\right) \delta$ $215.3(\mathrm{C}), 138.1(\mathrm{C}), 135.9(\mathrm{CH}), 135.8(\mathrm{CH}), 134.1(\mathrm{C}), 133.4(\mathrm{C}), 129.8(\mathrm{CH}), 129.7(\mathrm{CH}), 128.3$ $(\mathrm{CH}), 127.7(\mathrm{CH}), 127.6(\mathrm{CH}), 127.5(\mathrm{CH}), 75.2(\mathrm{CH}), 73.3\left(\mathrm{CH}_{2}\right), 72.2\left(\mathrm{CH}_{2}\right), 71.4(\mathrm{CH}), 47.9(\mathrm{CH})$, $44.7(\mathrm{CH}), 27.0\left(\mathrm{CH}_{3}\right), 20.1\left(\mathrm{CH}_{3}\right), 19.3(\mathrm{C}), 14.1\left(\mathrm{CH}_{3}\right), 10.2\left(\mathrm{CH}_{3}\right)$. HRMS (+FAB): $\mathrm{m} / z$ calcd. for $\mathrm{C}_{32} \mathrm{H}_{43} \mathrm{O}_{4} \mathrm{Si}[\mathrm{M}+\mathrm{H}]^{+}:$519.2931. Found: 519.2939 . 


\section{Stereochemical proof for $4 a$}

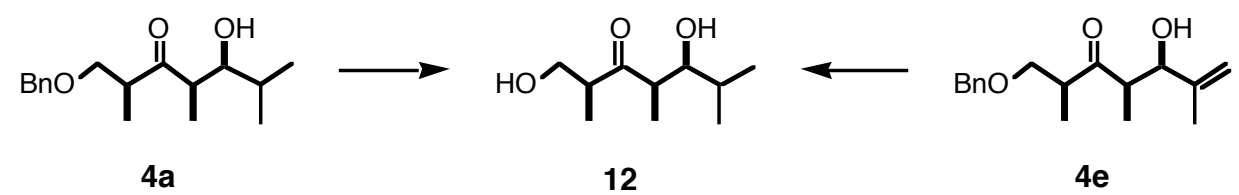

A mixture of $4 \mathbf{e}(56 \mathrm{mg}, 0.20 \mathrm{mmol})$ and $5 \% \mathrm{Pd} / \mathrm{C}(66 \mathrm{mg})$ in abs. EtOH $(2 \mathrm{~mL})$ was shaken overnight under $\mathrm{H}_{2}(1 \mathrm{~atm})$ at room temperature. The resulting suspension was filtered through a Celite $^{\circledR}$ pad, eluted with EtOAc, and concentrated in vacuo. A short flash chromatography $\left(\mathrm{CH}_{2} \mathrm{Cl}_{2} / \mathrm{MeOH} 95: 5\right)$ purification provided $35 \mathrm{mg}$ (>90\% yield) of (2S,4R,5S)-1,5-dihydroxy-2,4,6trimethyl-3-heptanone (12).

Following the same experimental procedure, hydrogenation of $\mathbf{4 a}(59 \mathrm{mg}, 0.21 \mathrm{mmol})$ afforded $\mathbf{1 2}$ in $92 \%$ yield.

$(2 S, 4 R, 5 S)-1,5-D i h y d r o x y-2,4,6$-trimethyl-3-heptanone (12). ${ }^{4}$ Colorless oil. $R_{f}\left(\mathrm{CH}_{2} \mathrm{Cl}_{2} / \mathrm{MeOH}\right.$ 95:5) $=0.30 ;[\alpha]_{\mathrm{D}}=-4.1$ (c 1.45, abs EtOH); IR (film): v 3416 (br), 2966, 2938, 2878, 1703, 1461, 1382, $1092 \mathrm{~cm}^{-1} ;{ }^{1} \mathrm{H}$ NMR (400 MHz, DMSO-d $)_{6} \delta 4.70(1 \mathrm{H}$, br t, $J \sim 5.2), 4.33(1 \mathrm{H}, \mathrm{br} \mathrm{d}, J \sim 6.0)$, 3.55 (1H, ddd, $J=10.4, J=7.1, J=5.2), 3.48$ (1H, q, $J=6.0), 3.35-3.27(1 \mathrm{H}, \mathrm{m}), 2.95-2.85(1 \mathrm{H}$, m), 2.81-2.73 (1H, m), 1.59-1.46 (1H, m), $0.96(3 \mathrm{H}, \mathrm{d}, J=6.8), 0.93(3 \mathrm{H}, \mathrm{d}, J=6.9), 0.86(3 \mathrm{H}, \mathrm{d}, J$ $=6.6), 0.83(3 \mathrm{H}, \mathrm{d}, J=6.7) ;{ }^{13} \mathrm{C} \mathrm{NMR}\left(100.6 \mathrm{MHz}, \mathrm{DMSO}-d_{6}\right) \delta 216.1(\mathrm{C}), 74.9(\mathrm{CH}), 63.3\left(\mathrm{CH}_{2}\right)$, $48.6(\mathrm{CH}), 46.4(\mathrm{CH}), 30.7(\mathrm{CH}), 19.9\left(\mathrm{CH}_{3}\right), 17.6\left(\mathrm{CH}_{3}\right), 13.8\left(\mathrm{CH}_{3}\right), 10.8\left(\mathrm{CH}_{3}\right)$. 


\section{Stereochemical proof for 11}

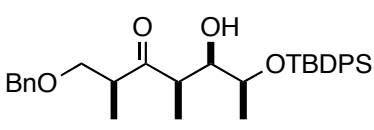

11

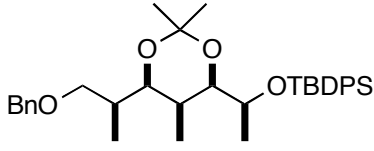

13

A 1.0 M THF solution of $\mathrm{Et}_{2} \mathrm{BOMe}(0.7 \mathrm{~mL}, 0.7 \mathrm{mmol})$ was added to a solution of aldol 11 (214 $\mathrm{mg}$, $0.41 \mathrm{mmol})$ in $\mathrm{THF}(3 \mathrm{~mL})$ at $-78{ }^{\circ} \mathrm{C}$. The resulting mixture was stirred at this temperature for 20 min and $\mathrm{NaBH}_{4}(19 \mathrm{mg}, 0.5 \mathrm{mmol})$ was added. Stirring continued for $3 \mathrm{~h}$ at $-78{ }^{\circ} \mathrm{C}$ and the reaction was quenched by addition of acetic acid $(0.8 \mathrm{~mL})$. The resulting mixture was diluted with $\mathrm{CH}_{2} \mathrm{Cl}_{2}$, washed with $\mathrm{H}_{2} \mathrm{O}$ and $1 \mathrm{M} \mathrm{NaOH}$ solution, dried $\left(\mathrm{MgSO}_{4}\right)$ and concentrated.

The resulting oil was diluted with $1 \mathrm{M} \mathrm{NaOAc}$ solution in $\mathrm{MeOH} / \mathrm{H}_{2} \mathrm{O} 9: 1(11 \mathrm{~mL})$ at $0{ }^{\circ} \mathrm{C}$ and $33 \%$ $\mathrm{H}_{2} \mathrm{O}_{2}(1.65 \mathrm{~mL})$ was added. The reacting mixture was stirred for $5 \mathrm{~min}$ at $0{ }^{\circ} \mathrm{C}$ and $1 \mathrm{~h}$ at room temperature, diluted with $\mathrm{CH}_{2} \mathrm{Cl}_{2}$, washed with $\mathrm{H}_{2} \mathrm{O}$, dried $\left(\mathrm{MgSO}_{4}\right)$ and concentrated in vacuo. The resulting oil was purified by flash chromatography (from 10\% to 30\% EtOAc in hexanes) on silica gel, which provided $85 \mathrm{mg}(0.16 \mathrm{mmol}, 40 \%$ yield $)$ of anti diol $\left(R_{f}\right.$ (hexanes/EtOAc 70:30) $\left.=0.40\right)$ and $108 \mathrm{mg}(0.21 \mathrm{mmol}, 50 \%$ yield $)$ of the desired syn diol $\left(R_{f}(\right.$ hexanes/EtOAc 70:30 $\left.)=0.35\right)$.

A solution of the desired syn diol and a catalytic amount of PPTS in $\mathrm{CH}_{2} \mathrm{Cl}_{2} / \mathrm{Me}_{2} \mathrm{C}(\mathrm{OMe})_{2}$ 1:1 (5 mL) was stirred at room temperature overnight. The mixture was concentrated and purified by flash chromatography $\left(\mathrm{CH}_{2} \mathrm{Cl}_{2}\right)$ on silica gel, which afforded $108 \mathrm{mg}(0.19 \mathrm{mmol}$, 93\% yield) of (2S,3R,4S,5R,6S)-1-benzyloxy-6-(tert-butyldiphenylsilyloxy)-3,5-O-isopropylidene-2,4-dimethyl3,5-heptanediol (13).

$(2 S, 3 R, 4 S, 5 R, 6 S)$-1-Benzyloxy-6-(tert-butyldiphenylsilyloxy)-3,5-O-isopropylidene-2,4-dimethyl3,5-heptanediol (13): colorless oil. $R_{f}\left(\mathrm{CH}_{2} \mathrm{Cl}_{2}\right)=0.55$; $[\alpha]_{\mathrm{D}}=-3.3\left(c\right.$ 1.05, $\left.\mathrm{CHCl}_{3}\right)$; IR (film): $v$

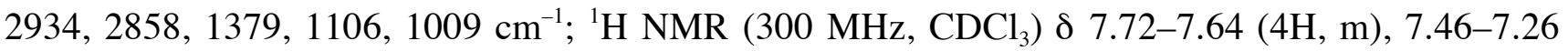
$(11 \mathrm{H}, \mathrm{m}), 4.52(1 \mathrm{H}, \mathrm{AB}$ system, $J=12.2), 4.45(1 \mathrm{H}, \mathrm{AB}$ system, $J=12.2), 3.70(1 \mathrm{H}, \mathrm{dq}, J=8.7, J=$ 5.9), 3.66 (1H, dd, $J=9.6, J=2.0), 3.59$ (1H, dd, $J=8.7, J=2.1), 3.41(1 \mathrm{H}, \mathrm{dd}, J=9.2, J=3.8)$, $3.33(1 \mathrm{H}, \mathrm{dd}, J=9.2, J=5.7), 2.07-1.98(1 \mathrm{H}, \mathrm{m}), 1.95-1.78(1 \mathrm{H}, \mathrm{m}), 1.37$ (3H, s), $1.33(3 \mathrm{H}, \mathrm{s}), 1.06$ $(3 \mathrm{H}, \mathrm{d}, J=6.6), 1.03(3 \mathrm{H}, \mathrm{d}, J=5.9), 1.01(9 \mathrm{H}, \mathrm{s}), 0.75(3 \mathrm{H}, \mathrm{d}, J=6.7) ;{ }^{13} \mathrm{C}$ NMR $(75.4 \mathrm{MHz}$, $\left.\mathrm{CDCl}_{3}\right) \delta 138.6(\mathrm{C}), 135.9(\mathrm{CH}), 135.9(\mathrm{CH}), 134.9(\mathrm{C}), 133.5(\mathrm{C}), 129.7(\mathrm{CH}), 129.4(\mathrm{CH}), 128.3$ $(\mathrm{CH}), 127.6(\mathrm{CH}), 127.5(\mathrm{CH}), 127.3(\mathrm{CH}), 99.0(\mathrm{C}), 78.4(\mathrm{CH}), 76.0(\mathrm{CH}), 73.2\left(\mathrm{CH}_{2}\right), 71.6\left(\mathrm{CH}_{2}\right)$, 
$68.8(\mathrm{CH}), 35.0(\mathrm{CH}), 30.2(\mathrm{CH}), 29.9\left(\mathrm{CH}_{3}\right), 27.0\left(\mathrm{CH}_{3}\right), 21.1\left(\mathrm{CH}_{3}\right), 19.7\left(\mathrm{CH}_{3}\right), 19.3(\mathrm{C}), 14.8$ $\left(\mathrm{CH}_{3}\right), 5.5\left(\mathrm{CH}_{3}\right)$.

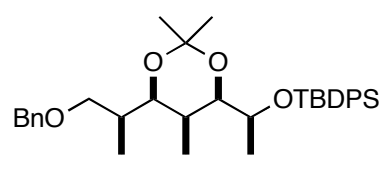

13

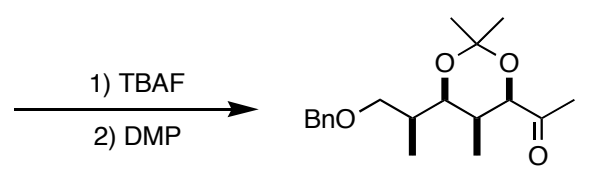

14

A solution of $13(49 \mathrm{mg}, 87.5 \mu \mathrm{mol})$ and TBAF $(2.1 \mathrm{mmol})$ in THF $(2.1 \mathrm{~mL})$ was stirred overnight at room temperature. The reaction mixture was diluted with $\mathrm{CH}_{2} \mathrm{Cl}_{2}$, washed with $\mathrm{H}_{2} \mathrm{O}$ and brine, dried $\left(\mathrm{MgSO}_{4}\right)$ and concentrated. Purification of the resulting oil by flash chromatography (hexanes/EtOAc $70: 30)$ on silica gel afforded $27 \mathrm{mg}(84 \mu \mathrm{mol}, 95 \%$ yield) of $(2 S, 3 R, 4 S, 5 R, 6 S)$-7-benzyloxy-3,5isopropylidenedioxy-4,6-dimethyl-2-heptanol, which was immediately used in the next step.

A mixture of the abovementioned alcohol (27 mg, $84 \mu \mathrm{mol}), \mathrm{NaHCO}_{3}(105 \mathrm{mg}, 1.25 \mathrm{mmol})$ and DMP $(73 \mathrm{mg}, 167 \mu \mathrm{mol})$ in non-dried $\mathrm{CH}_{2} \mathrm{Cl}_{2}(0.85 \mathrm{~mL})$ was stirred at $0{ }^{\circ} \mathrm{C}$ for one day. The reaction mixture was diluted with $\mathrm{CH}_{2} \mathrm{Cl}_{2}$, washed with sat $\mathrm{NaHCO}_{3}$, dried $\left(\mathrm{Na}_{2} \mathrm{SO}_{4}\right)$ and concentrated. Purification of the resulting oil by flash chromatography (hexanes/EtOAc 90:10) afforded $23 \mathrm{mg}$ (72 $\mu \mathrm{mol}, 86 \%$ yield) of $(3 R, 4 S, 5 R, 6 S)$-7-benzyloxy-3,5-isopropylidenedioxy-4,6-dimethyl-2-heptanone (14).

(3R,4S,5R,6S)-7-Benzyloxy-3,5-isopropylidenedioxy-4,6-dimethyl-2-heptanone (14): ${ }^{5,6}$ colorless viscous oil. $R_{f}$ (hexanes/EtOAc 90:10) $=0.2 ;[\alpha]_{\mathrm{D}}=+32.9\left(c 1.0, \mathrm{CHCl}_{3}\right)\left[\right.$ lit. $^{5}[\alpha]_{\mathrm{D}}=+33.6(c$ 2.0, $\left.\mathrm{CHCl}_{3}\right)$; lit. $\left.{ }^{6}[\alpha]_{\mathrm{D}}=+30\left(c 1.0, \mathrm{CHCl}_{3}\right)\right]$; IR (film): $v$ 2991, 2932, 2857, 1717, 1455, 1384, 1356, 1202, 1159, 1109, $1016 \mathrm{~cm}^{-1} ;{ }^{1} \mathrm{H}$ NMR (300 MHz, $\left.\mathrm{CDCl}_{3}\right) \delta 7.40-7.25(5 \mathrm{H}, \mathrm{m}), 4.48(2 \mathrm{H}, \mathrm{s}), 4.25$ $(1 \mathrm{H}, \mathrm{d}, J=2.7), 3.71(1 \mathrm{H}, \mathrm{dd}, J=9.6, J=2.1), 3.36(1 \mathrm{H}, \mathrm{dd}, J=9.3, J=4.3), 3.32(1 \mathrm{H}, \mathrm{dd}, J=9.3, J$ $=5.1), 2.16(3 \mathrm{H}, \mathrm{s}), 2.07-1.97(1 \mathrm{H}, \mathrm{m}), 1.92-1.77(1 \mathrm{H}, \mathrm{m}), 1.48(3 \mathrm{H}, \mathrm{s}), 1.41(3 \mathrm{H}, \mathrm{s}), 1.05(3 \mathrm{H}, \mathrm{d}, J$ $=6.6), 0.81(3 \mathrm{H}, \mathrm{d}, J=6.6) ;{ }^{13} \mathrm{C} \mathrm{NMR}\left(75.4 \mathrm{MHz}, \mathrm{CDCl}_{3}\right) \delta 209.8(\mathrm{C}), 138.4(\mathrm{C}), 128.3(\mathrm{CH}), 127.6$ $(\mathrm{CH}), 127.5(\mathrm{CH}), 99.4(\mathrm{C}), 79.4(\mathrm{CH}), 75.2(\mathrm{CH}), 73.2\left(\mathrm{CH}_{2}\right), 71.3\left(\mathrm{CH}_{2}\right), 34.9(\mathrm{CH}), 32.4(\mathrm{CH})$, $29.8\left(\mathrm{CH}_{3}\right), 27.1\left(\mathrm{CH}_{3}\right), 19.2\left(\mathrm{CH}_{3}\right), 14.5\left(\mathrm{CH}_{3}\right), 6.4\left(\mathrm{CH}_{3}\right)$. 


\section{6. ${ }^{1} \mathrm{H}$ and ${ }^{13} \mathrm{C}$ spectra of aldol diastereomers, diol 12 and ketone 14}

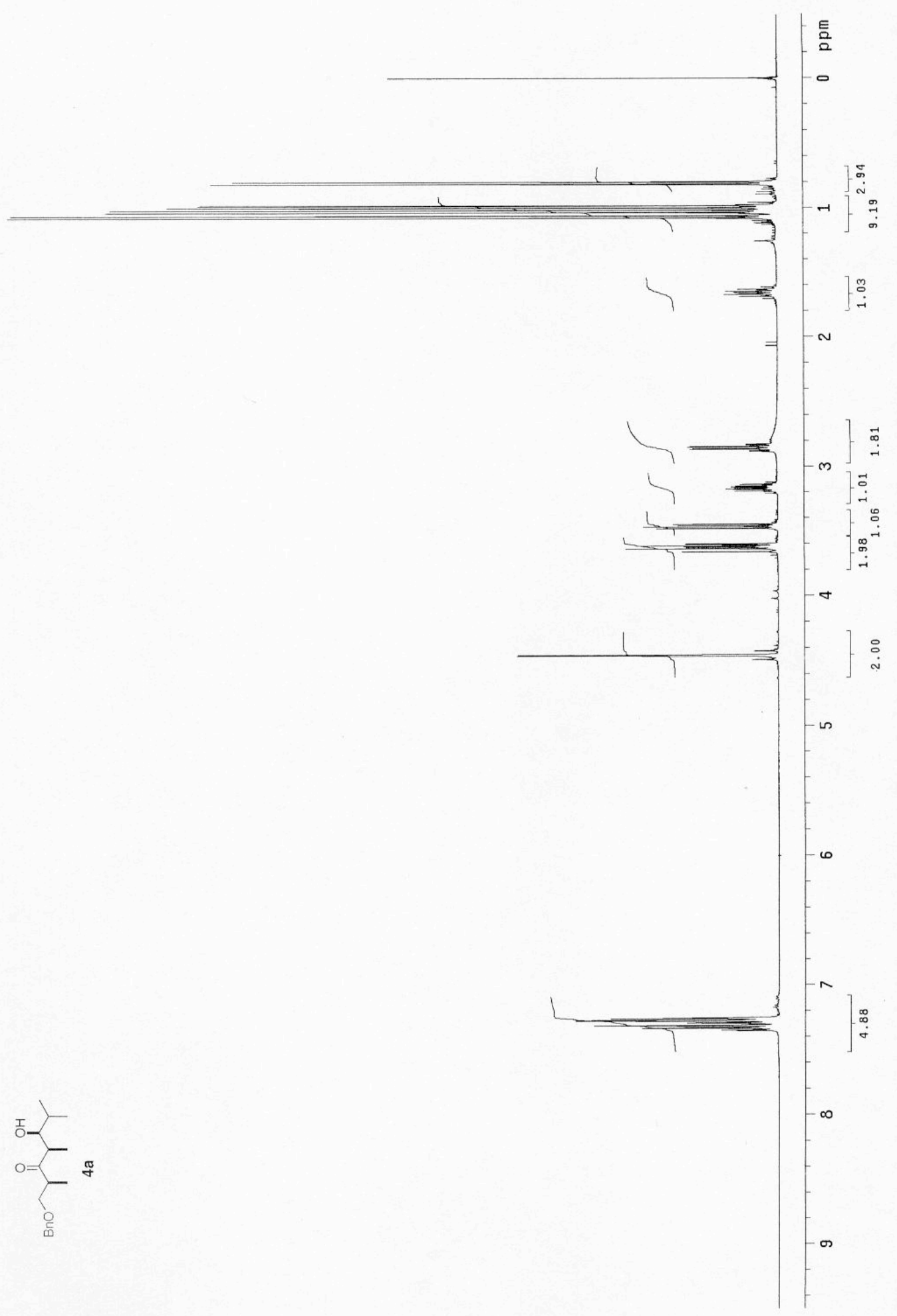




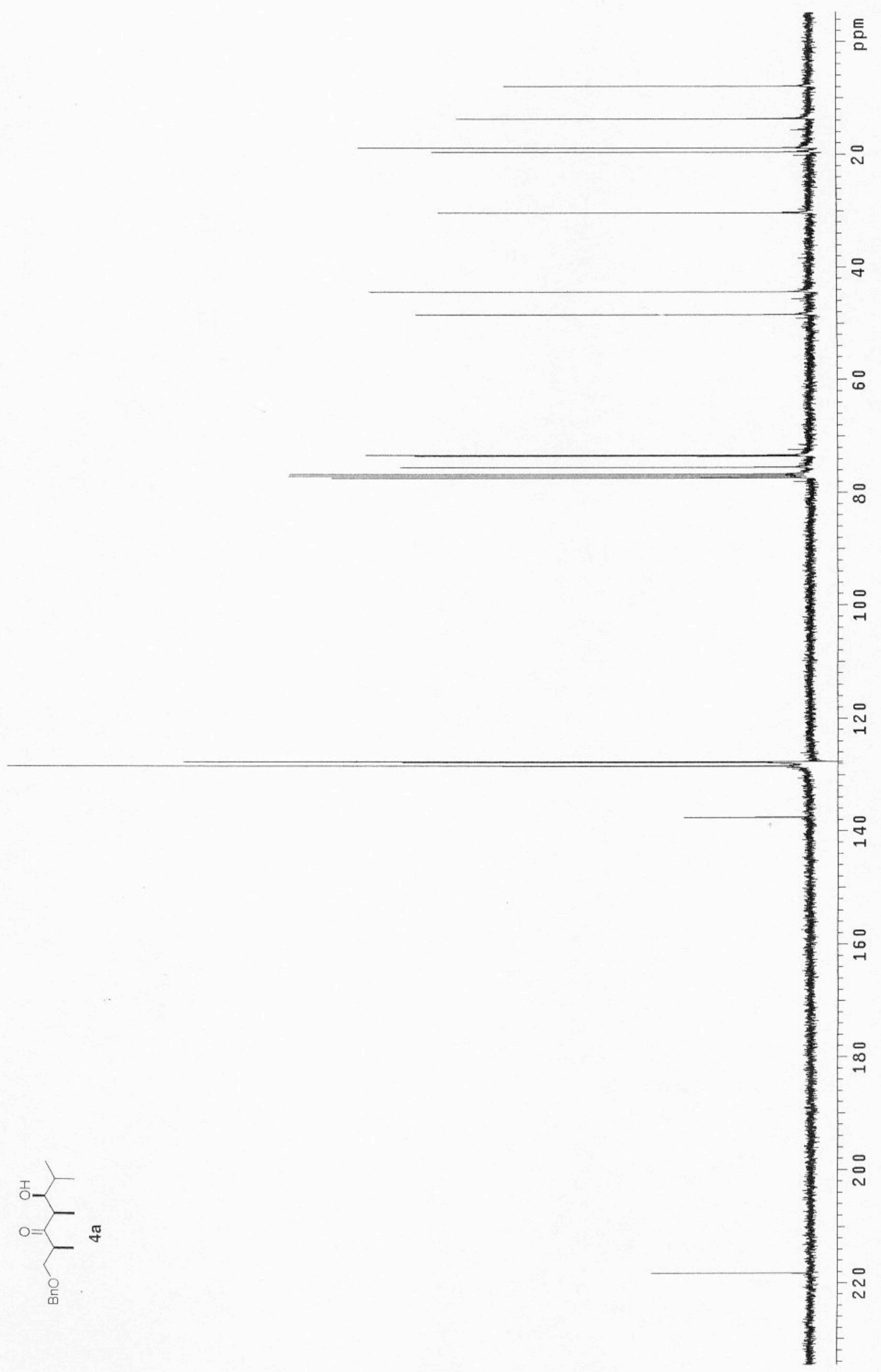




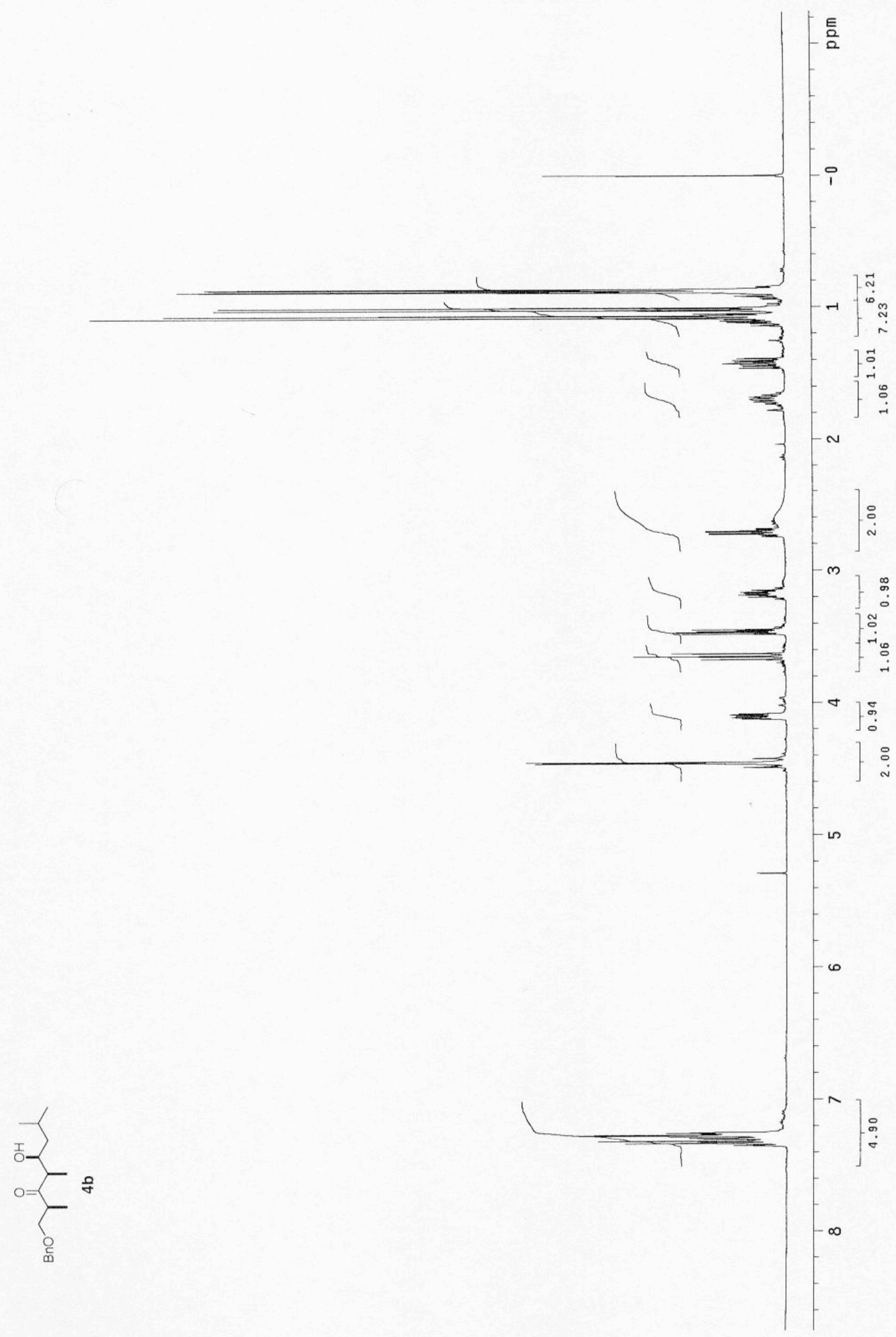




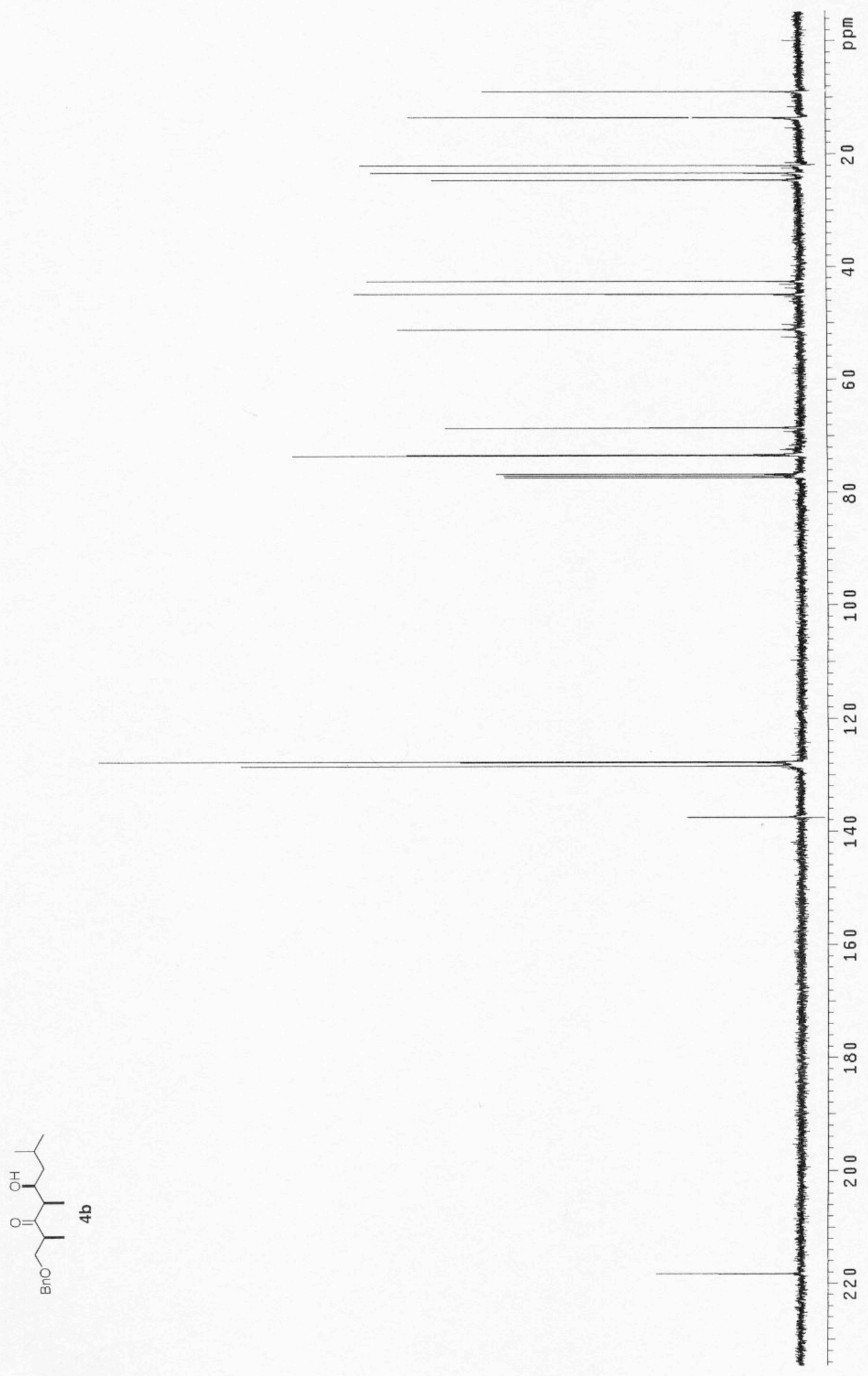


Highly Stereoselective Aldol Reaction Based on Titanium Enolates from (S)-1-Benzyloxy-2-methyl-3-pentanone. Joan G. Solsona, Joaquim Nebot, Pedro Romea, * Fèlix Urpí.*

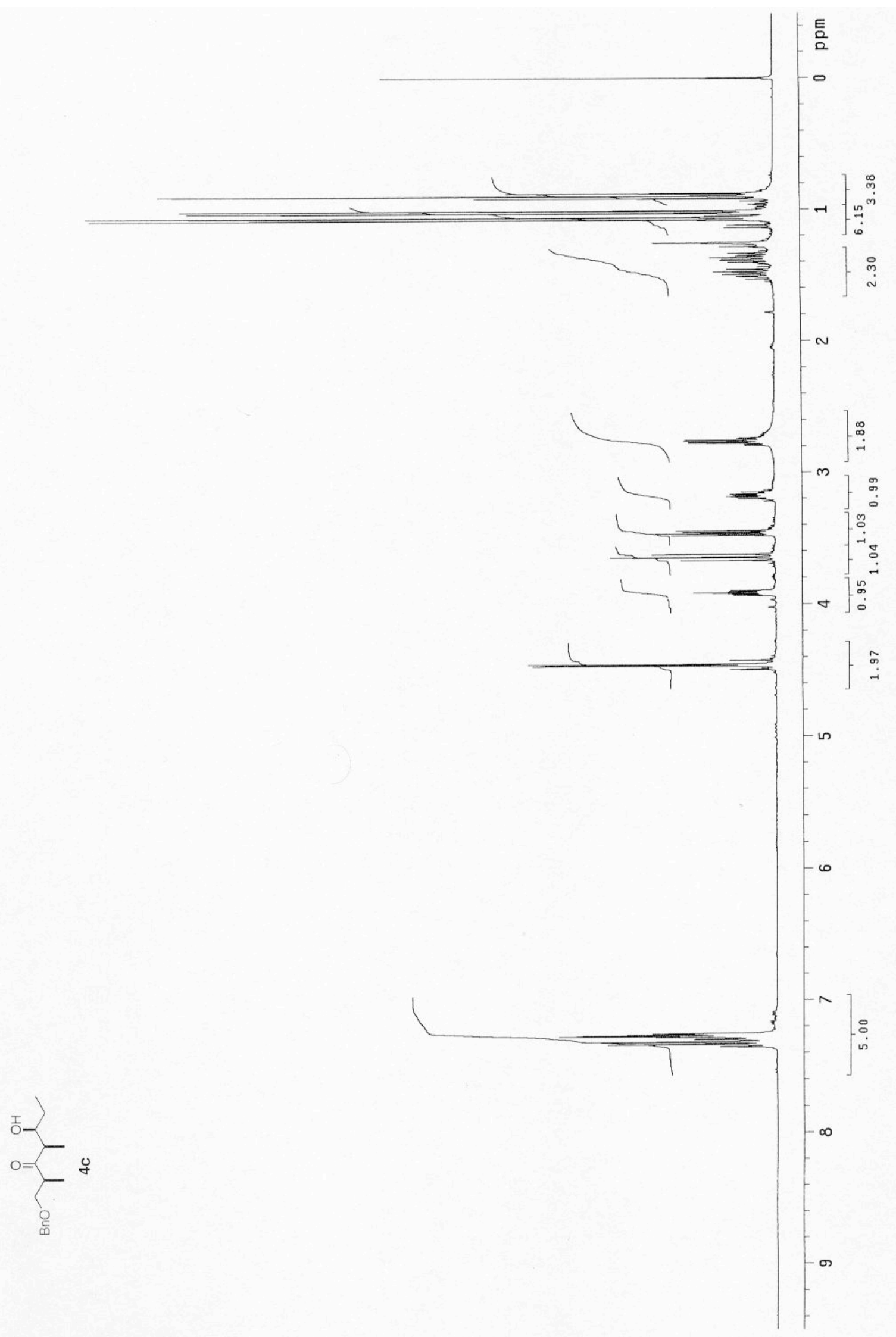


Highly Stereoselective Aldol Reaction Based on Titanium Enolates from (S)-1-Benzyloxy-2-methyl-3-pentanone. Joan G. Solsona, Joaquim Nebot, Pedro Romea, * Fèlix Urpí.*

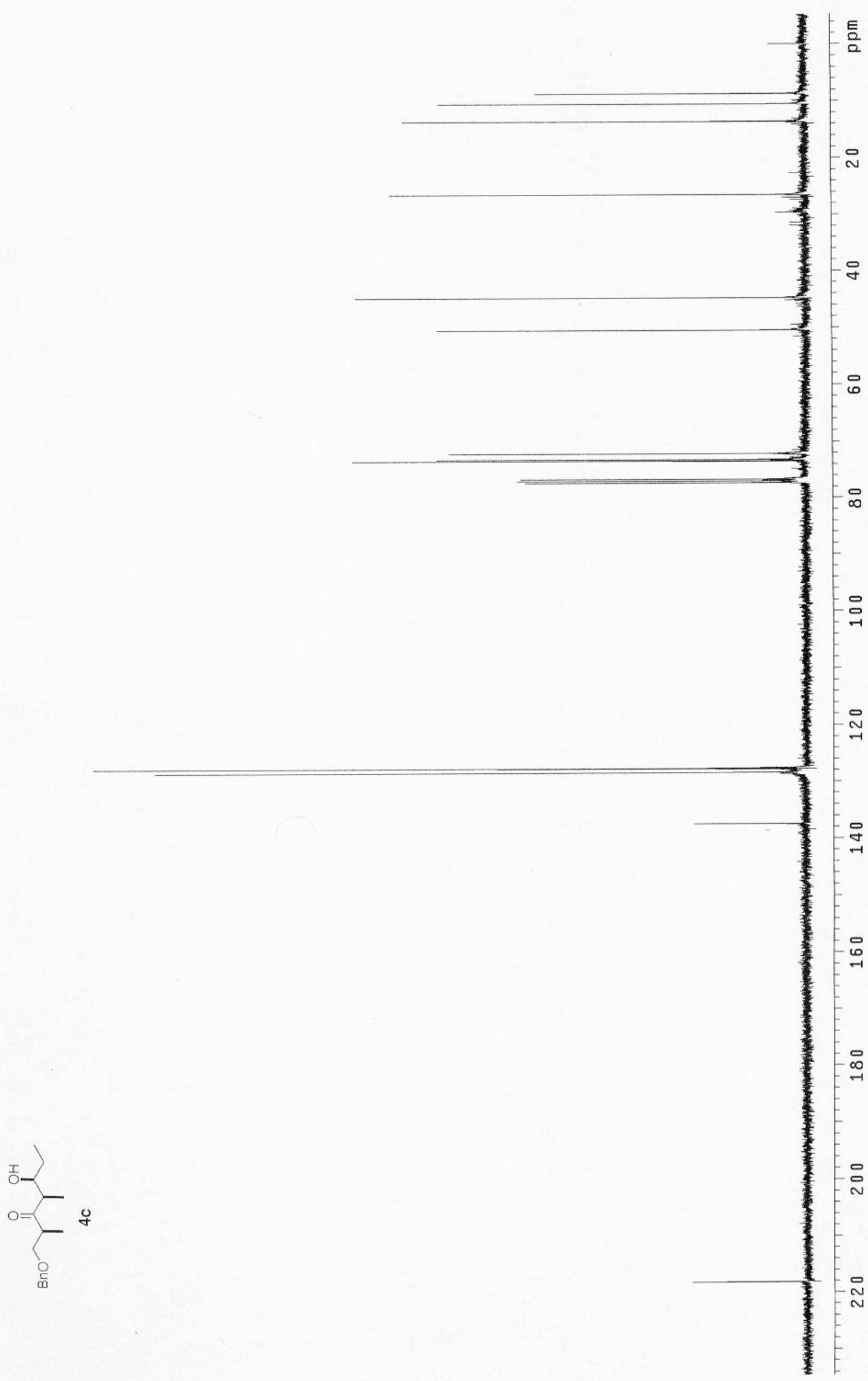


Highly Stereoselective Aldol Reaction Based on Titanium Enolates from (S)-1-Benzyloxy-2-methyl-3-pentanone. Joan G. Solsona, Joaquim Nebot, Pedro Romea, * Fèlix Urpí.*

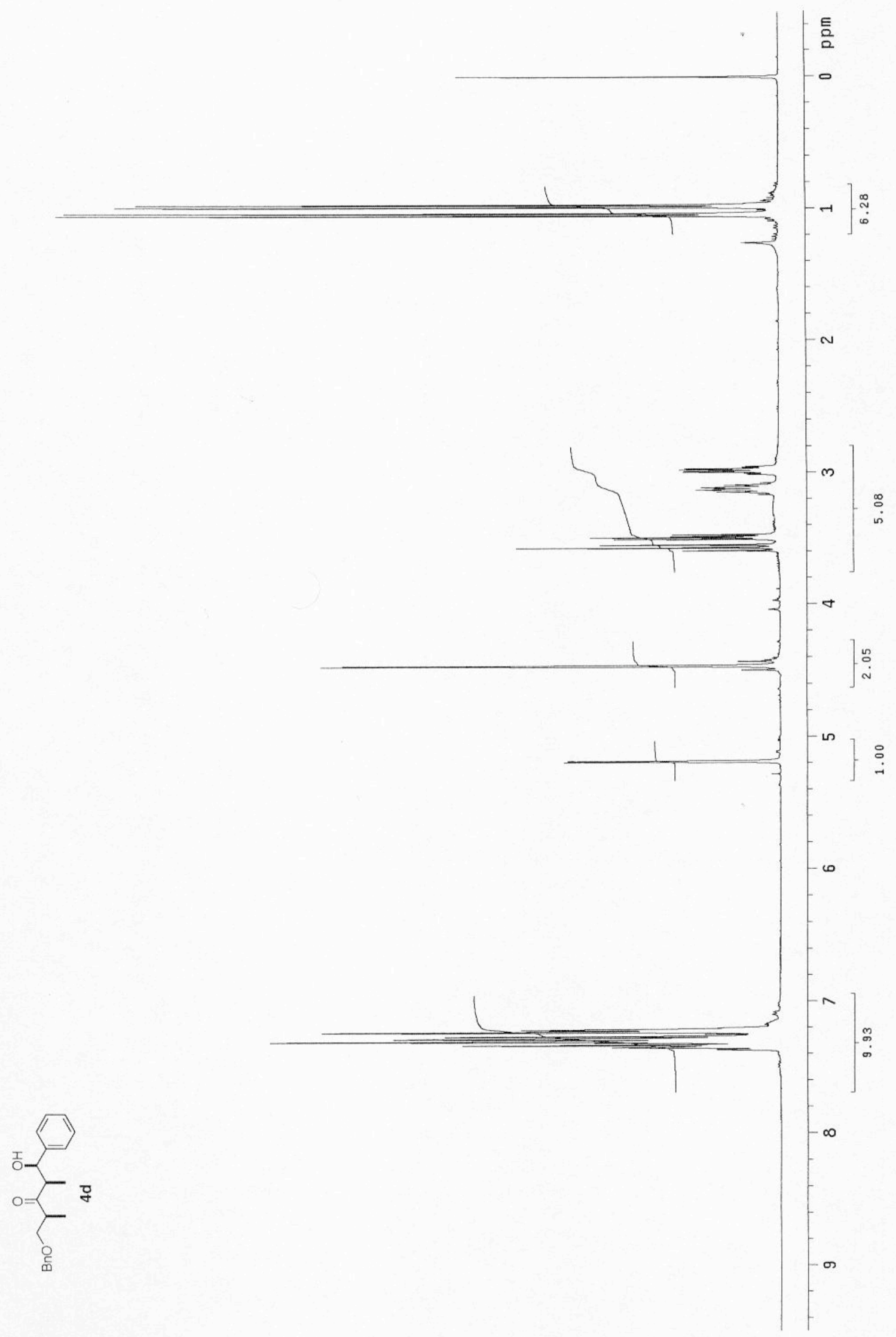


Highly Stereoselective Aldol Reaction Based on Titanium Enolates from (S)-1-Benzyloxy-2-methyl-3-pentanone. Joan G. Solsona, Joaquim Nebot, Pedro Romea, * Fèlix Urpí.*

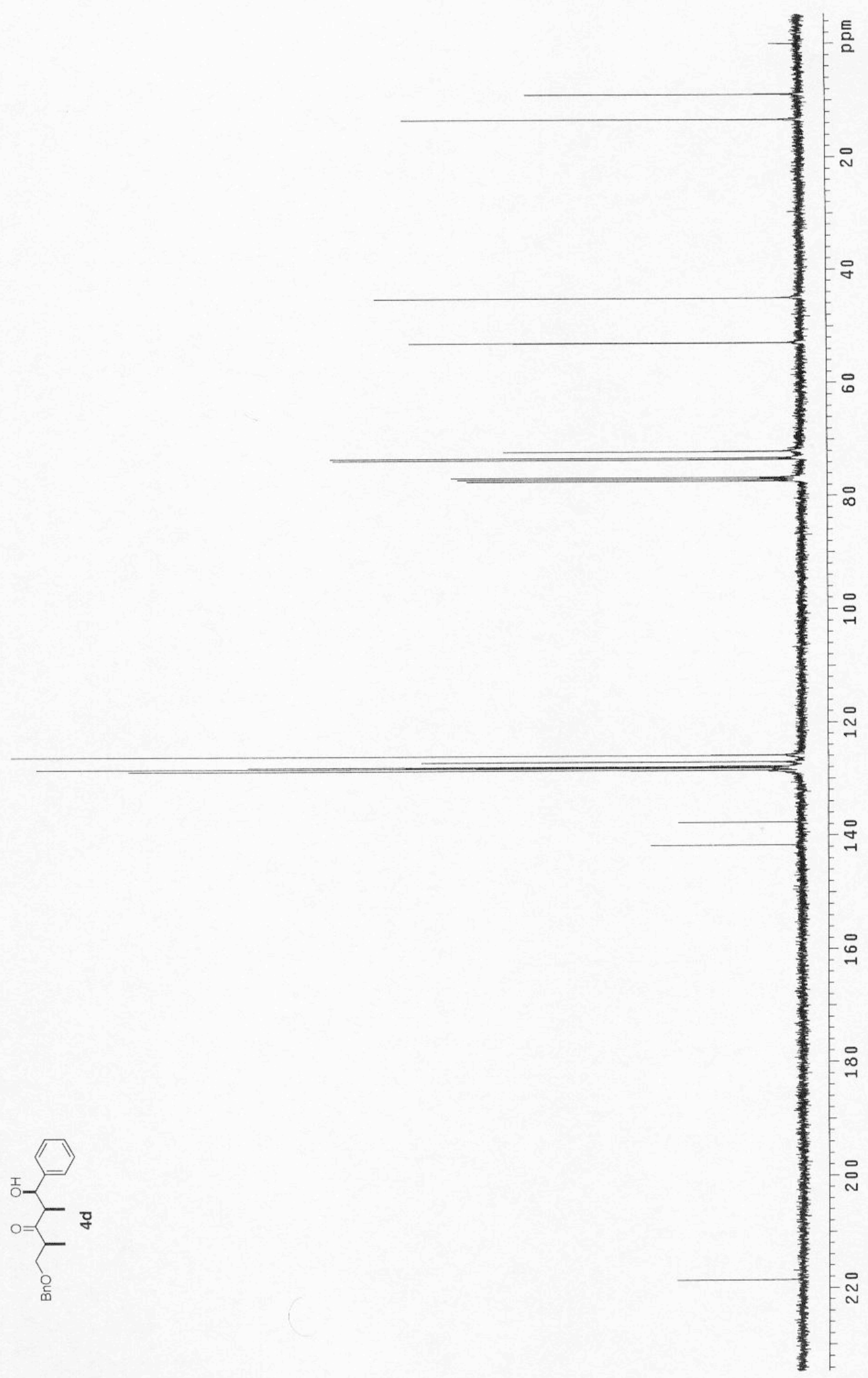


Highly Stereoselective Aldol Reaction Based on Titanium Enolates from (S)-1-Benzyloxy-2-methyl-3-pentanone. Joan G. Solsona, Joaquim Nebot, Pedro Romea, * Fèlix Urpí.*

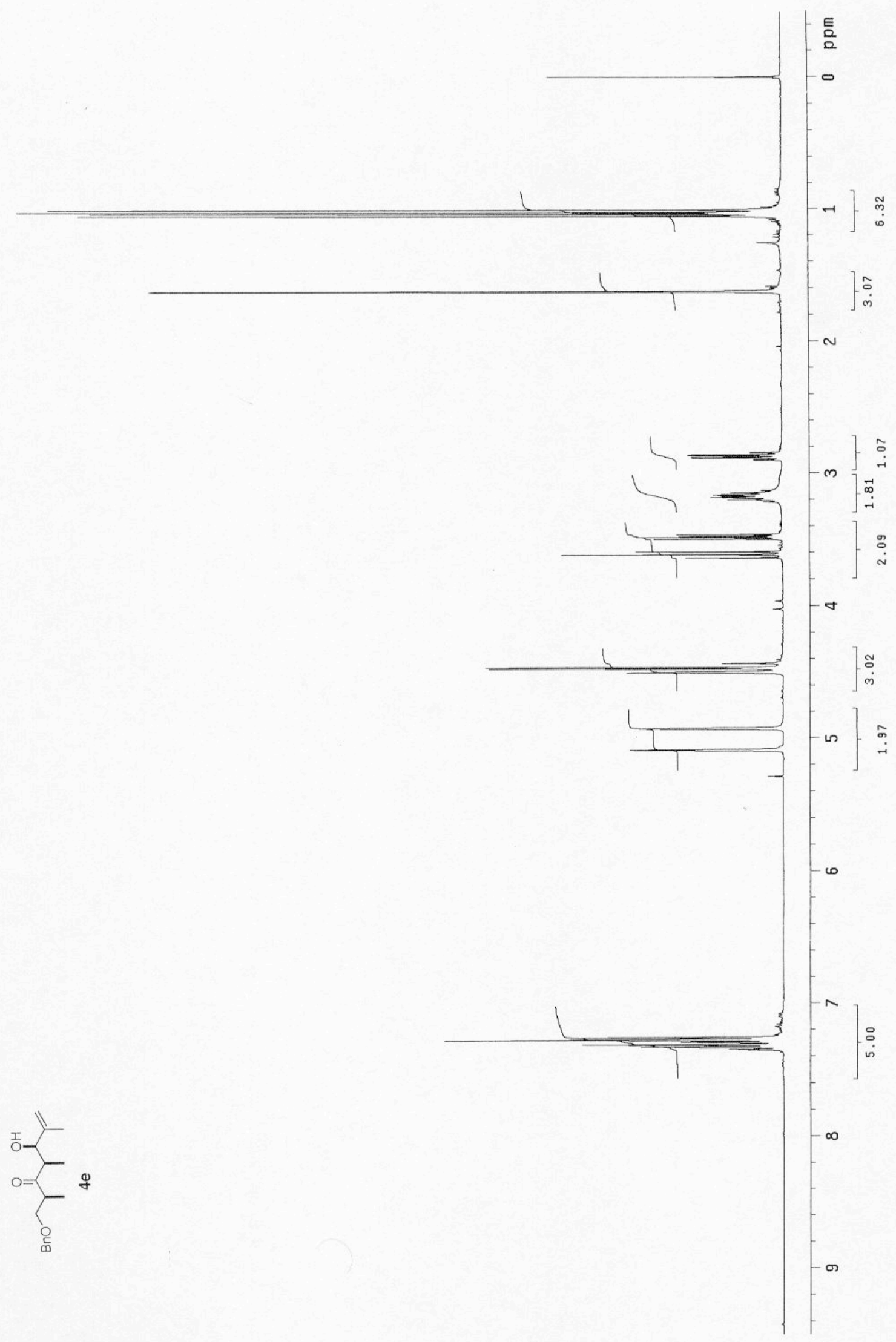


Highly Stereoselective Aldol Reaction Based on Titanium Enolates from (S)-1-Benzyloxy-2-methyl-3-pentanone.

Joan G. Solsona, Joaquim Nebot, Pedro Romea, * Fèlix Urpí.*

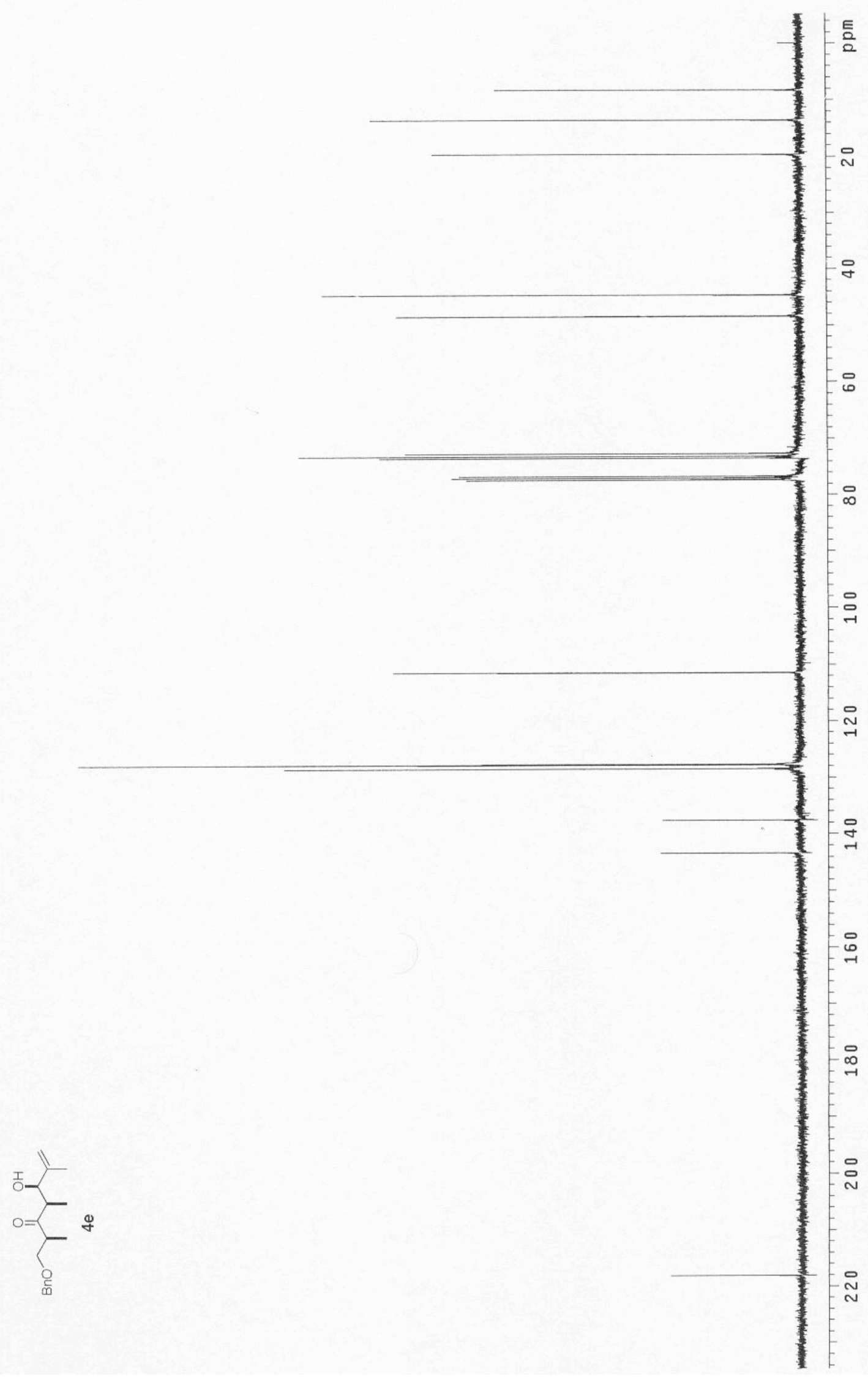


Highly Stereoselective Aldol Reaction Based on Titanium Enolates from (S)-1-Benzyloxy-2-methyl-3-pentanone. Joan G. Solsona, Joaquim Nebot, Pedro Romea, * Fèlix Urpí.*

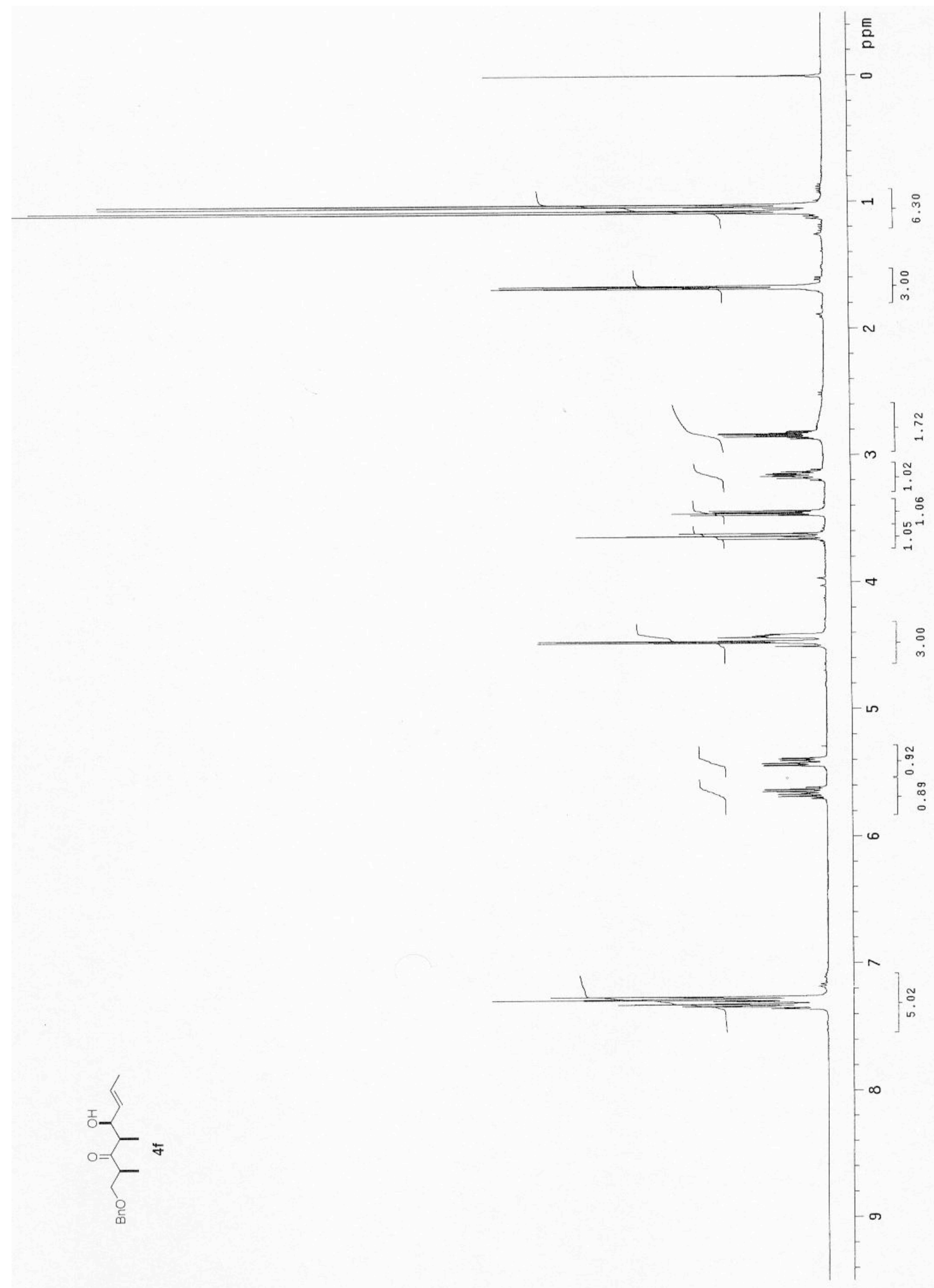


Highly Stereoselective Aldol Reaction Based on Titanium Enolates from (S)-1-Benzyloxy-2-methyl-3-pentanone. Joan G. Solsona, Joaquim Nebot, Pedro Romea, * Fèlix Urpí.*

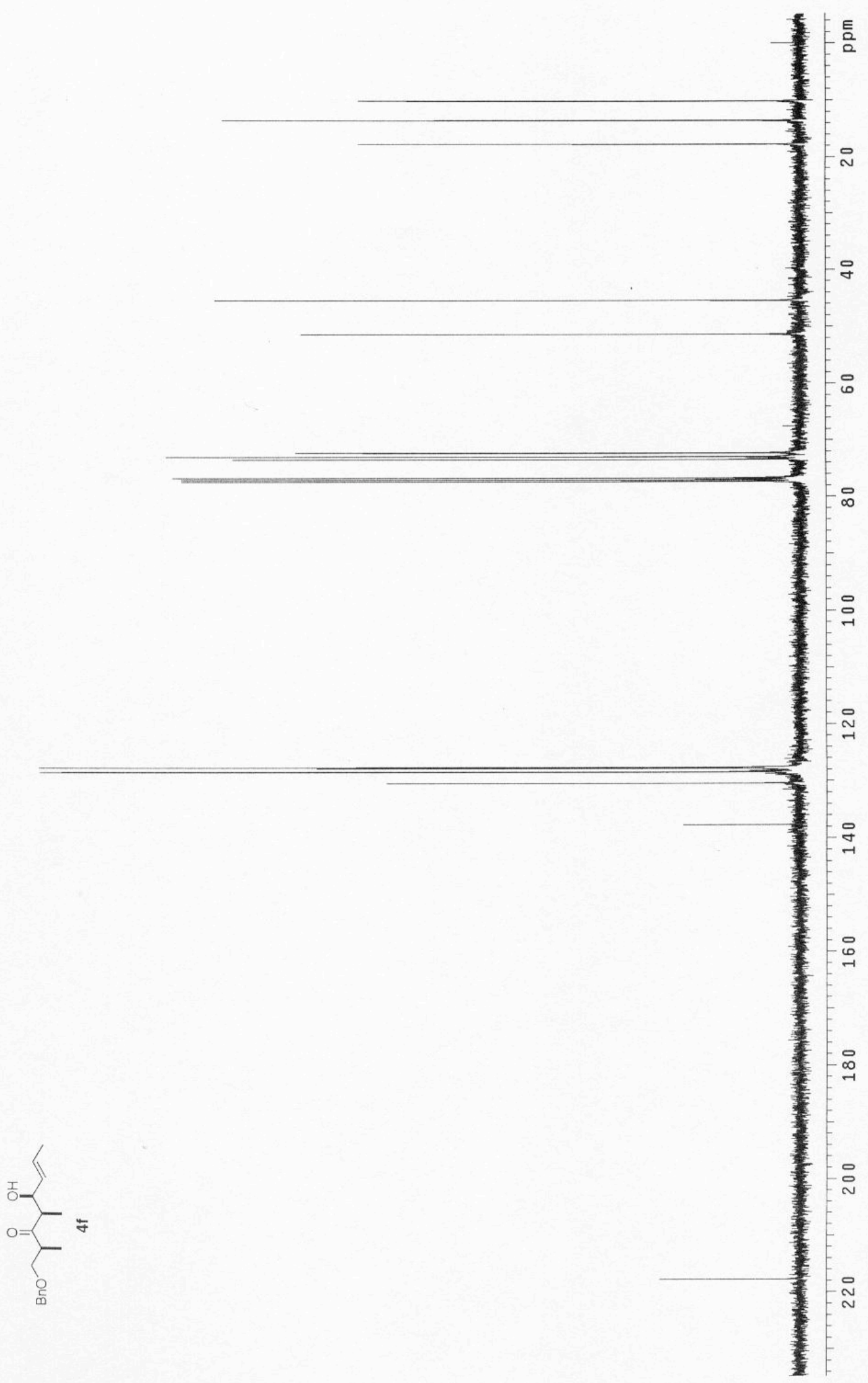


Highly Stereoselective Aldol Reaction Based on Titanium Enolates from (S)-1-Benzyloxy-2-methyl-3-pentanone. Joan G. Solsona, Joaquim Nebot, Pedro Romea, * Fèlix Urpí.*

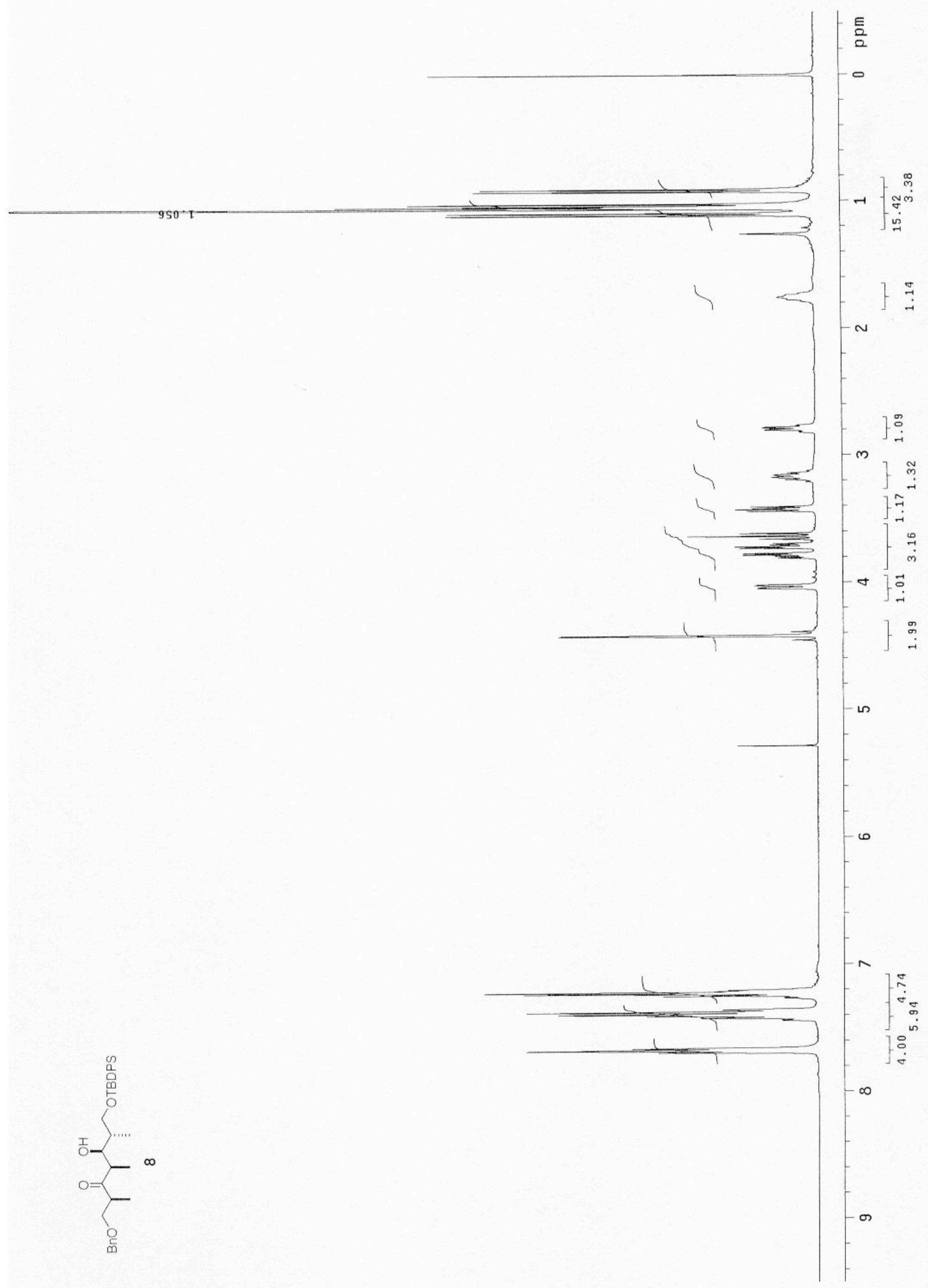


Highly Stereoselective Aldol Reaction Based on Titanium Enolates from (S)-1-Benzyloxy-2-methyl-3-pentanone. Joan G. Solsona, Joaquim Nebot, Pedro Romea, * Fèlix Urpí.*

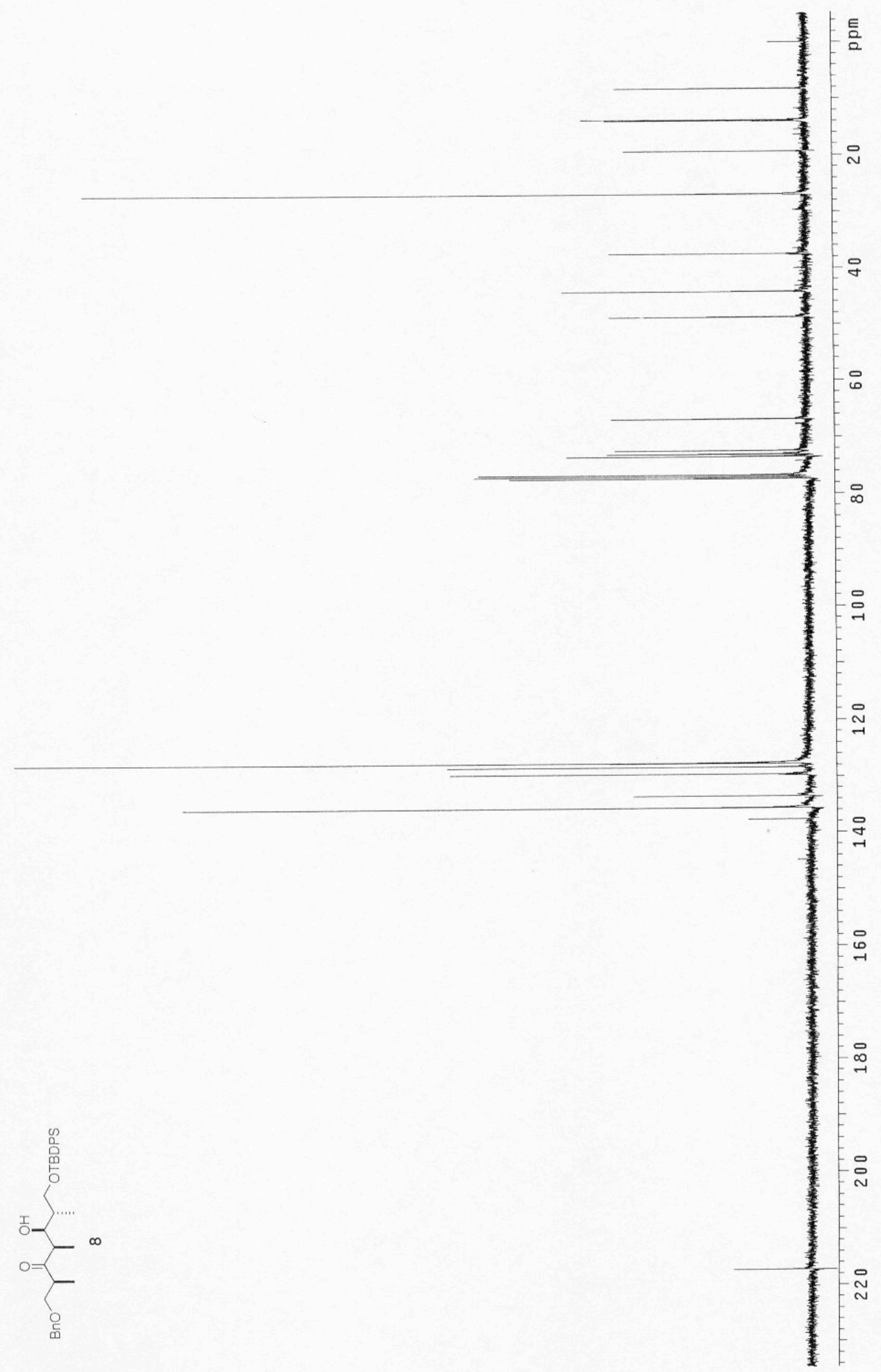


Highly Stereoselective Aldol Reaction Based on Titanium Enolates from (S)-1-Benzyloxy-2-methyl-3-pentanone. Joan G. Solsona, Joaquim Nebot, Pedro Romea, * Fèlix Urpí.*

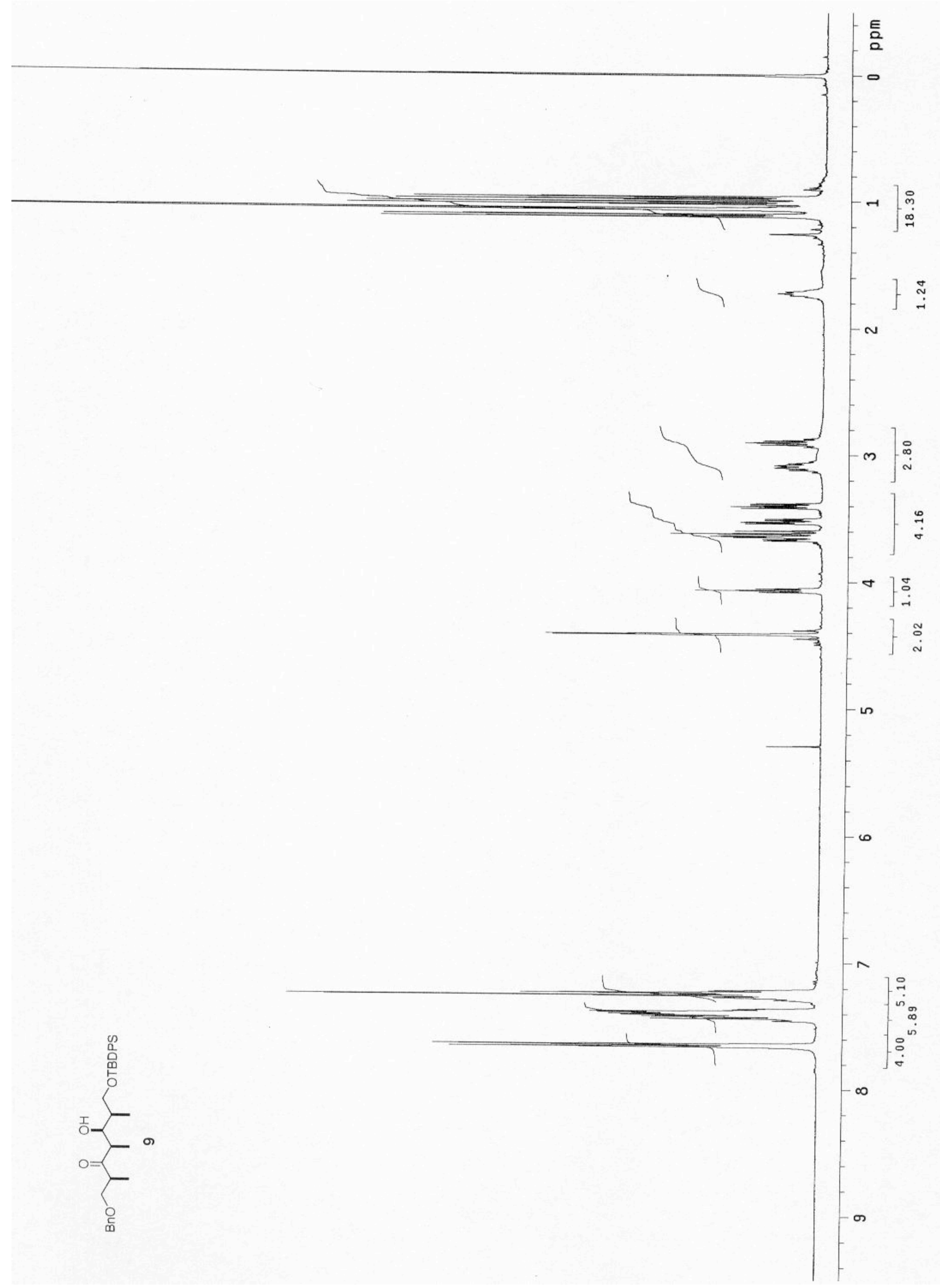


Highly Stereoselective Aldol Reaction Based on Titanium Enolates from (S)-1-Benzyloxy-2-methyl-3-pentanone. Joan G. Solsona, Joaquim Nebot, Pedro Romea, * Fèlix Urpí.*

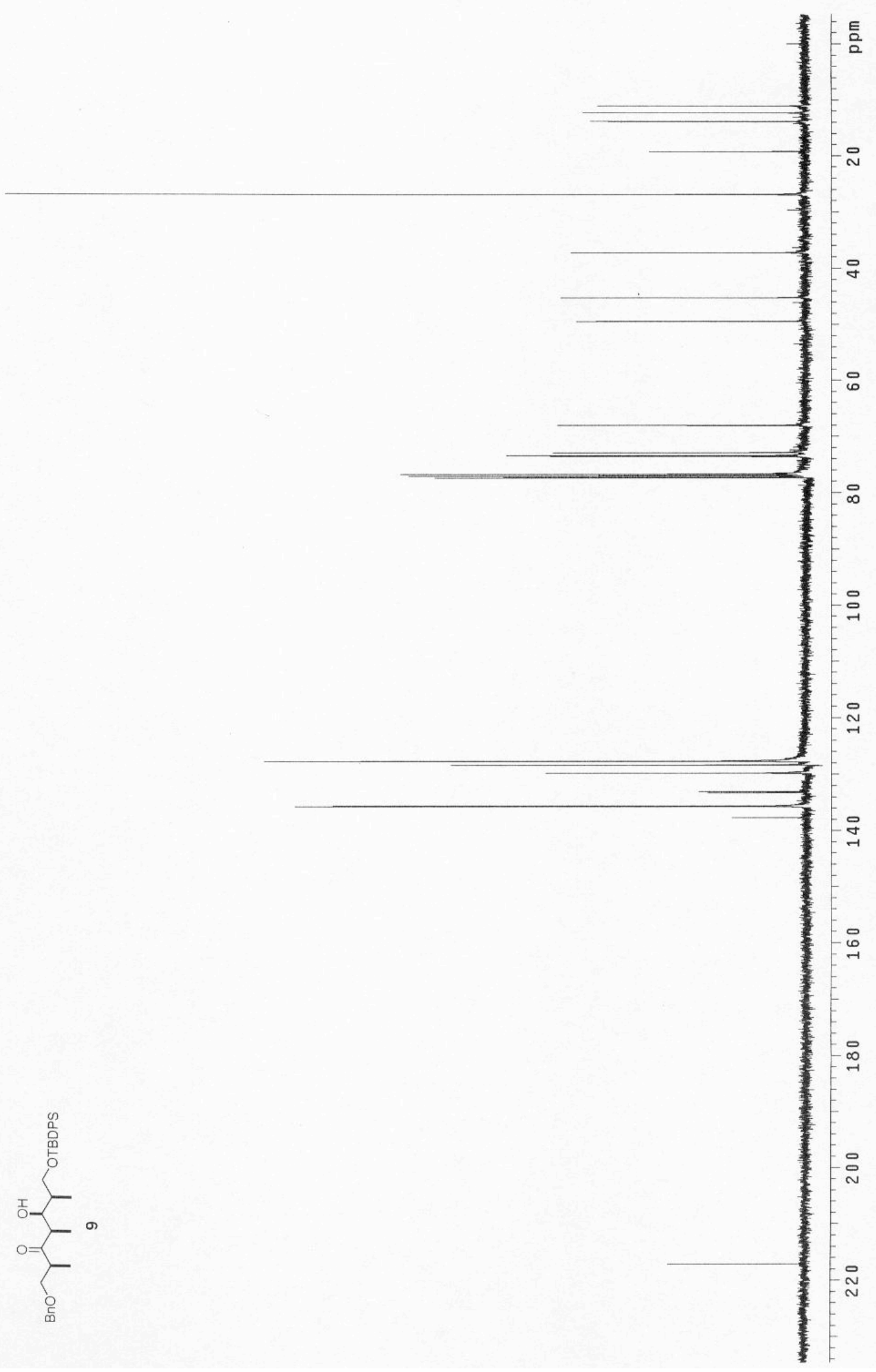


Highly Stereoselective Aldol Reaction Based on Titanium Enolates from (S)-1-Benzyloxy-2-methyl-3-pentanone. Joan G. Solsona, Joaquim Nebot, Pedro Romea, * Fèlix Urpí.*

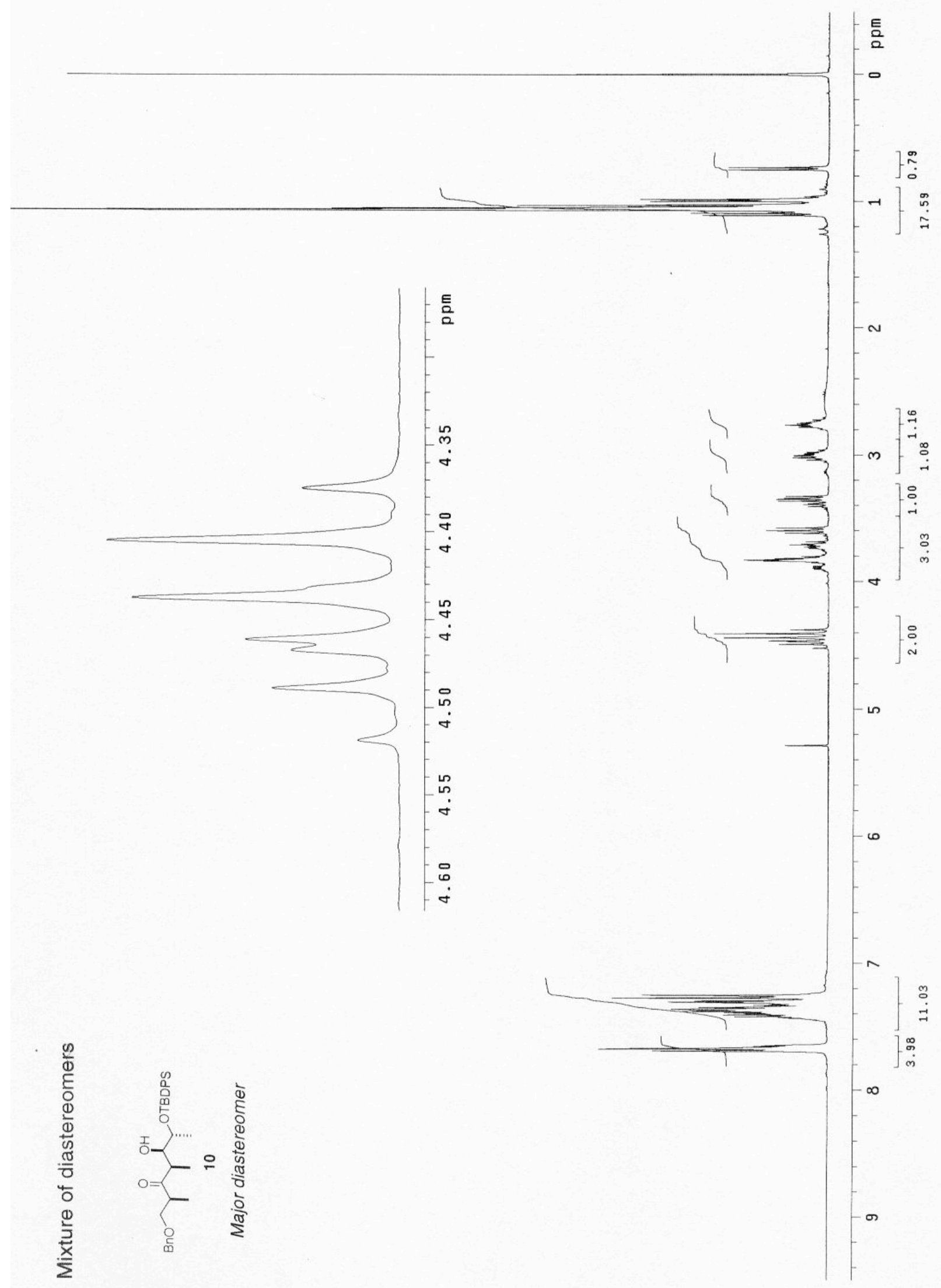


Highly Stereoselective Aldol Reaction Based on Titanium Enolates from (S)-1-Benzyloxy-2-methyl-3-pentanone. Joan G. Solsona, Joaquim Nebot, Pedro Romea, * Fèlix Urpí.*

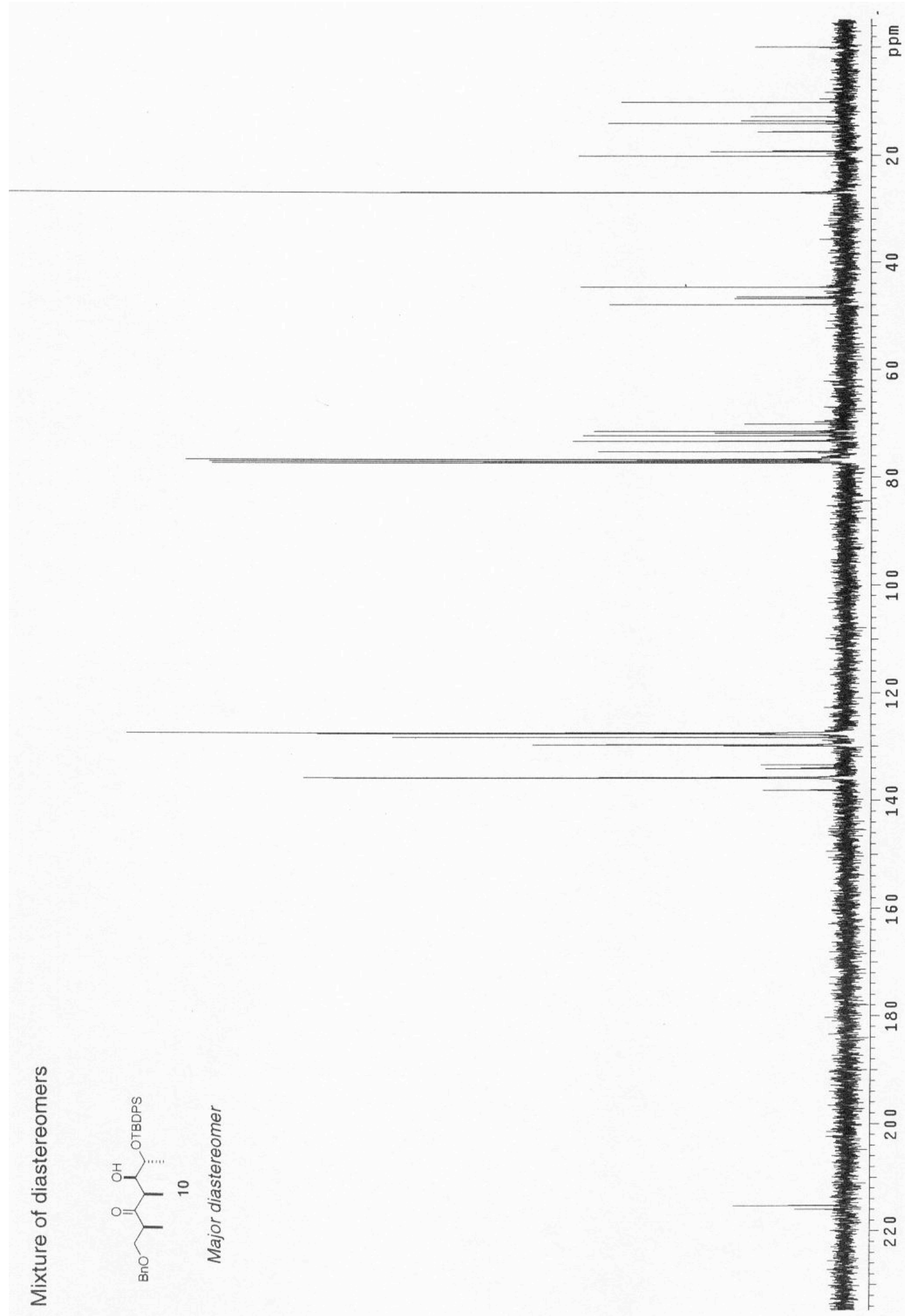


Highly Stereoselective Aldol Reaction Based on Titanium Enolates from (S)-1-Benzyloxy-2-methyl-3-pentanone.

Joan G. Solsona, Joaquim Nebot, Pedro Romea, * Fèlix Urpí.*

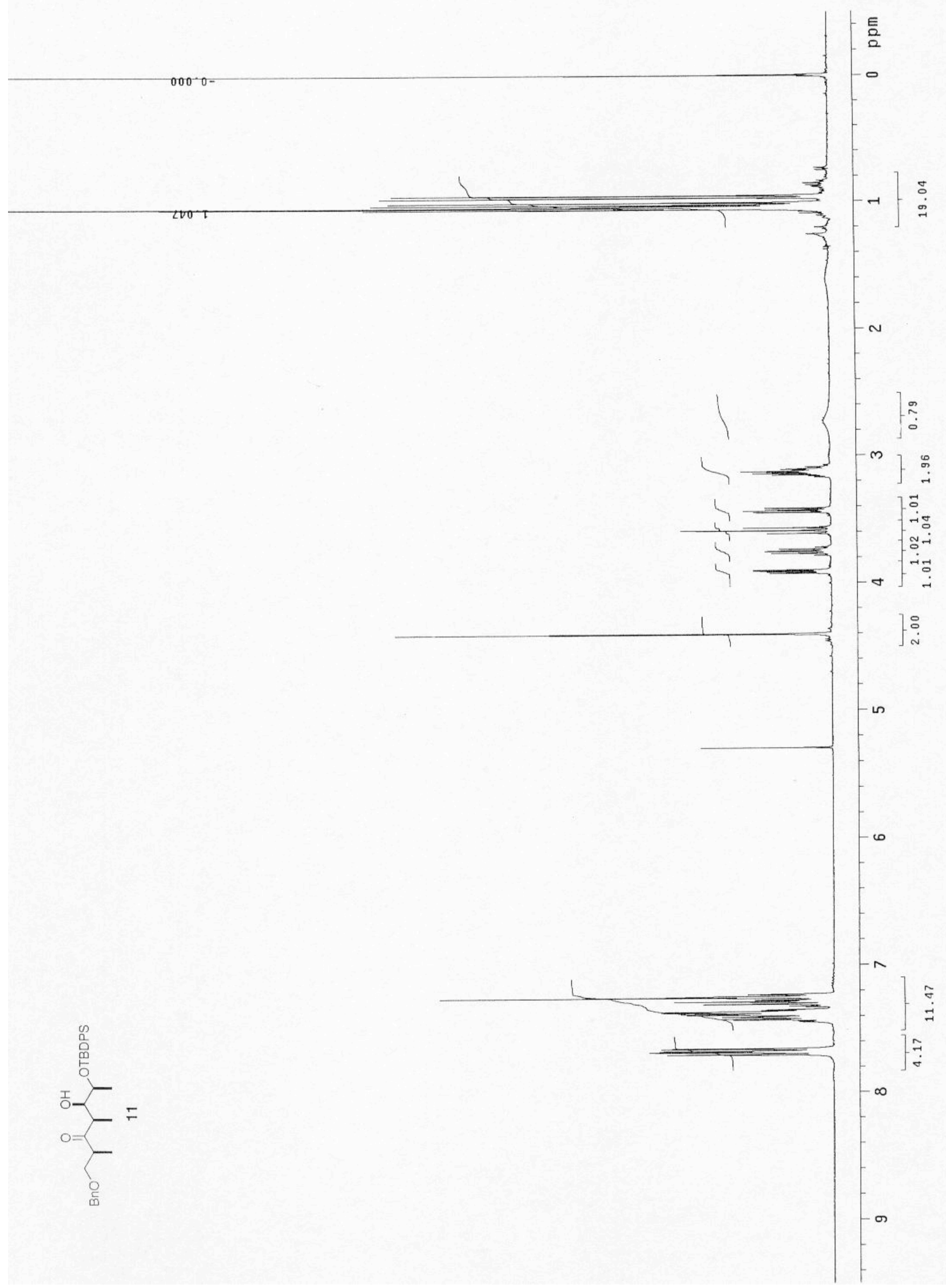


Highly Stereoselective Aldol Reaction Based on Titanium Enolates from (S)-1-Benzyloxy-2-methyl-3-pentanone. Joan G. Solsona, Joaquim Nebot, Pedro Romea, * Fèlix Urpí.*

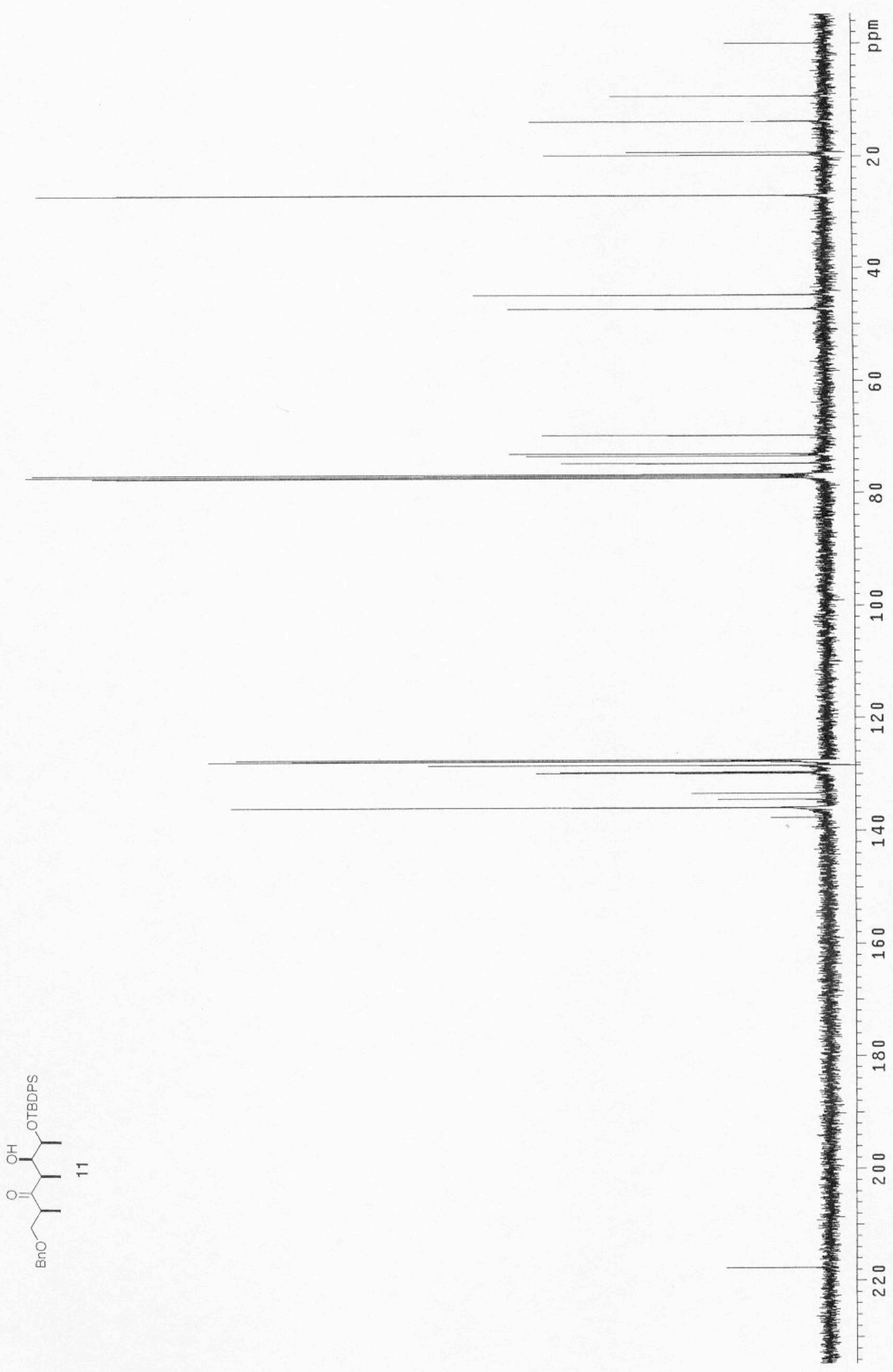


Highly Stereoselective Aldol Reaction Based on Titanium Enolates from (S)-1-Benzyloxy-2-methyl-3-pentanone. Joan G. Solsona, Joaquim Nebot, Pedro Romea, * Fèlix Urpí.*

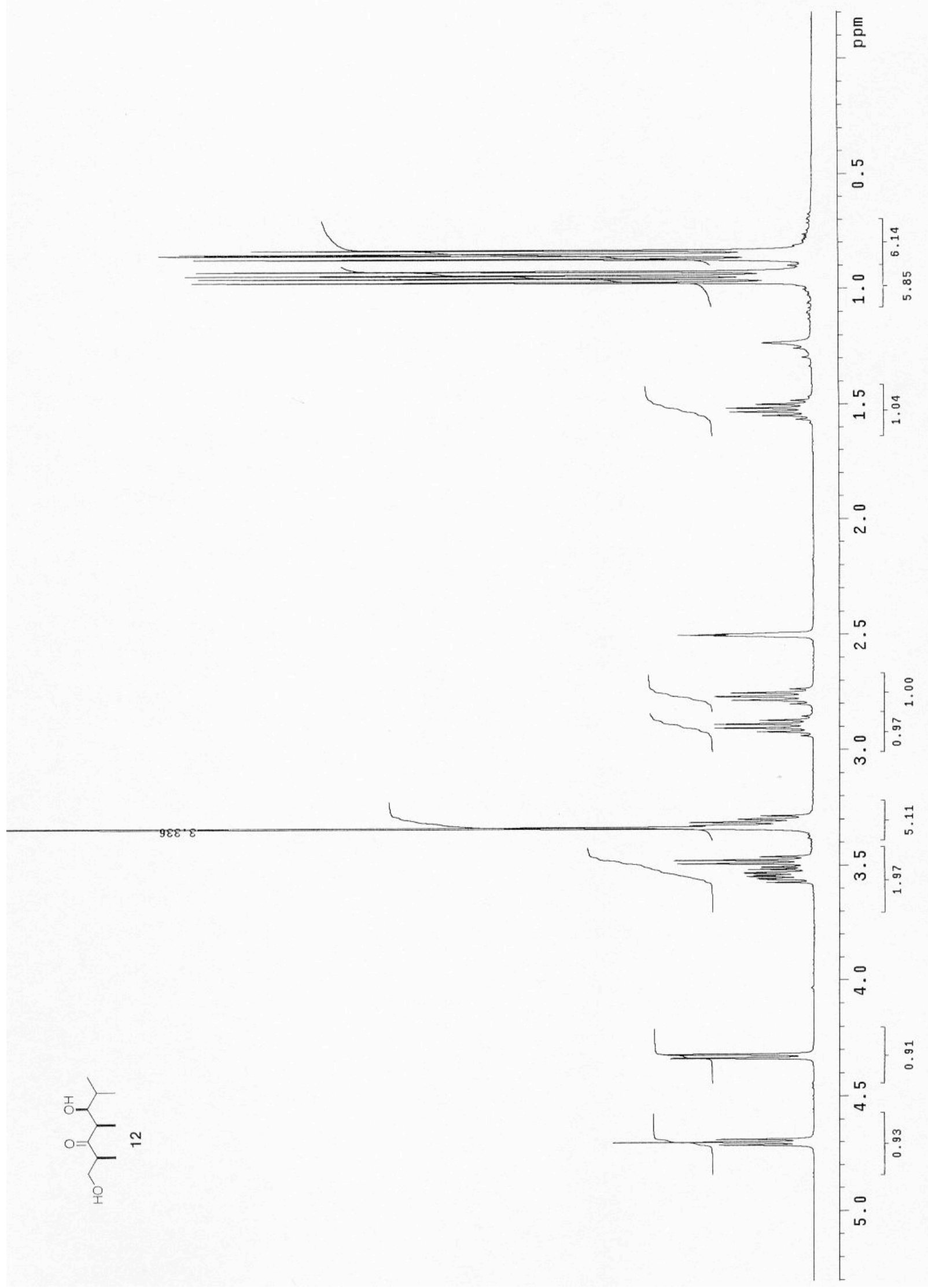


Highly Stereoselective Aldol Reaction Based on Titanium Enolates from (S)-1-Benzyloxy-2-methyl-3-pentanone. Joan G. Solsona, Joaquim Nebot, Pedro Romea, * Fèlix Urpí.*

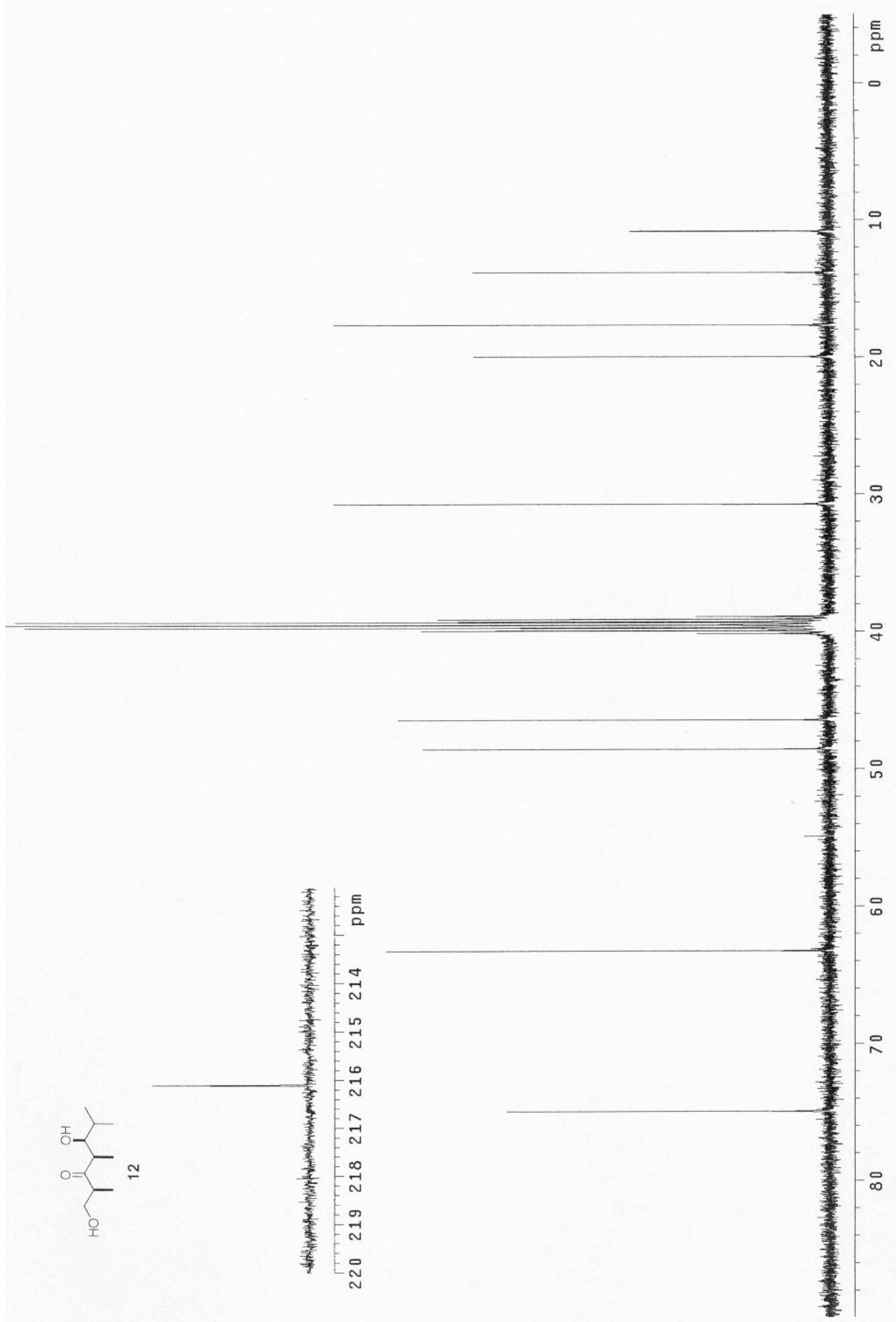




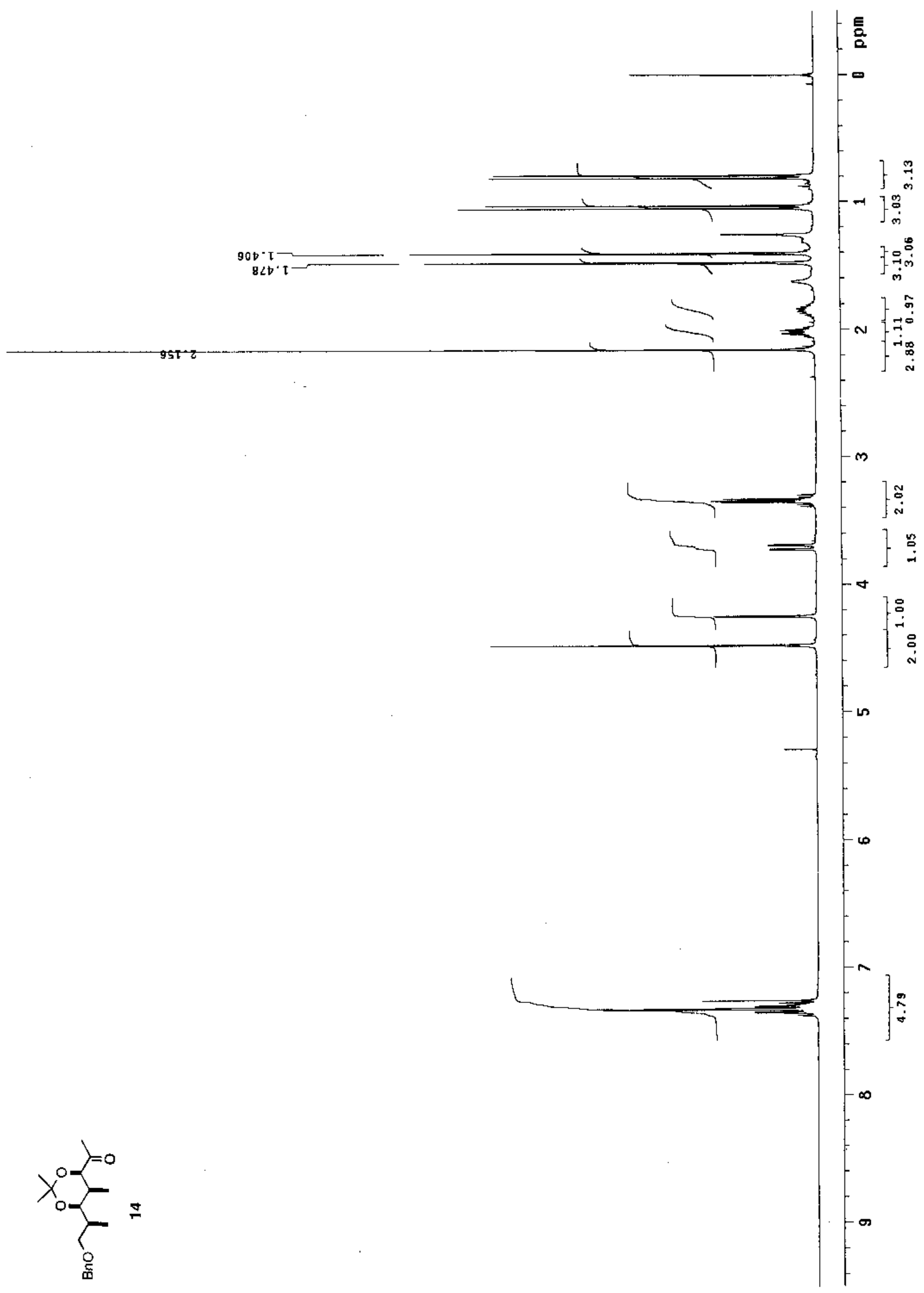


Highly Stereoselective Aldol Reaction Based on Titanium Enolates from (S)-1-Benzyloxy-2-methyl-3-pentanone. Joan G. Solsona, Joaquim Nebot, Pedro Romea, * Fèlix Urpí.*

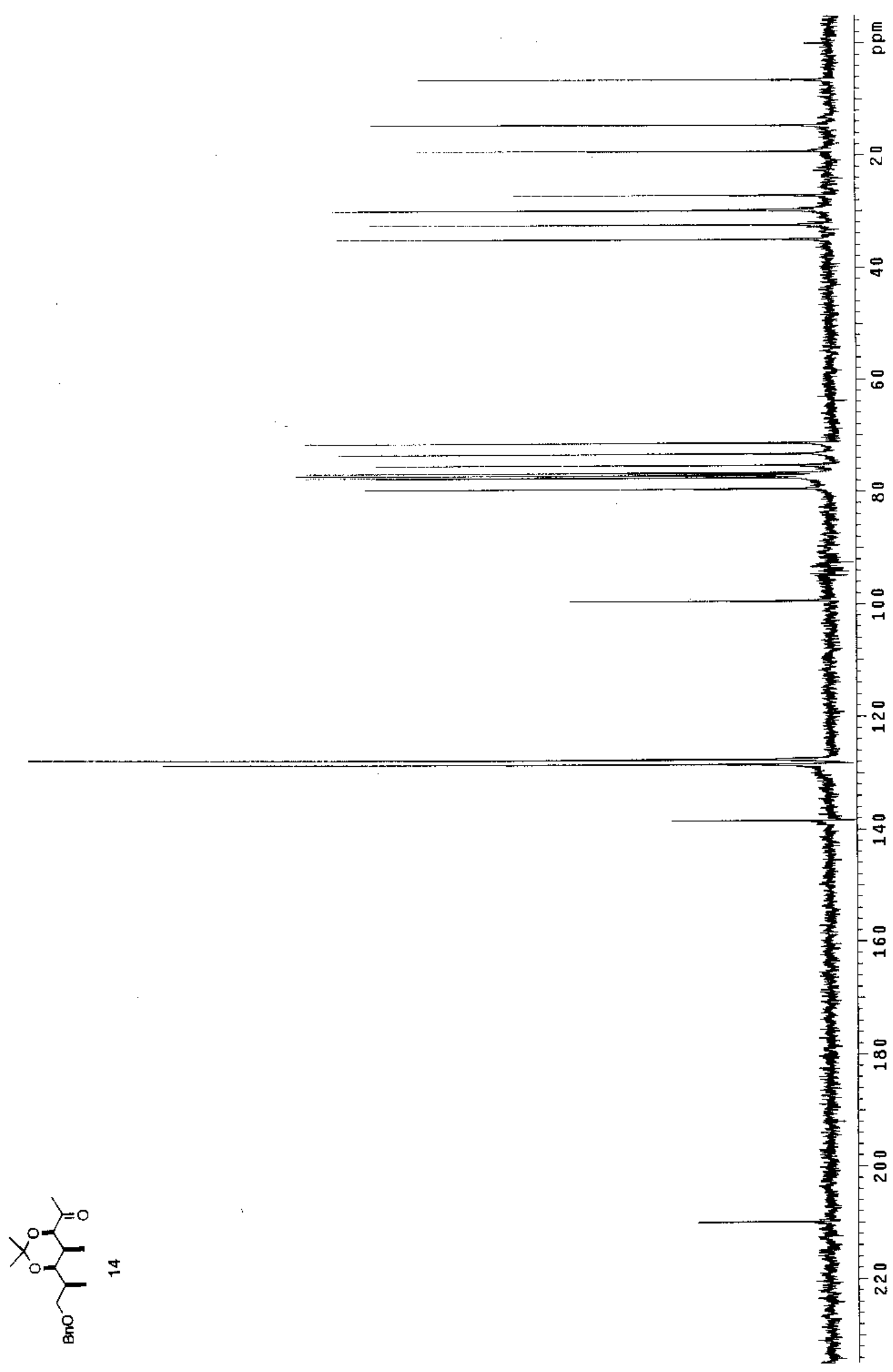


${ }^{1}$ Paterson, I.; Perkins, M. V. J. Am. Chem. Soc. 1993, 115, 1608-1610

${ }^{2}$ Paterson, I.; Norcross, R. D.; Ward, R. A.; Romea, P.; Lister, M. A. J. Am. Chem. Soc. 1994, 116, 11287-11314.

${ }^{3}$ Paterson, I.; Tillyer, R. D. Tetrahedron Lett. 1992, 33, 4233-4236.

${ }^{4}$ Luke G. P.; Morris, J. J. Org. Chem. 1985, 60, 3013-3019.

${ }^{5}$ Mulzer, J.; Kirstein, H. M.; Buschmann, J.; Lehmann, C.; Luger, P. J. Am. Chem. Soc. 1991, 113, 910-923.

${ }^{6}$ Gerber-Lemaire, S.; Ainge, S. W.; Glanzmann, C.; Vogel, P. Helv. Chim. Acta 2002, 85, 417-430. 